\title{
Intertwining Symmetry Algebras of Quantum Superintegrable Systems
}

\author{
Juan A. CALZADA ${ }^{\dagger}$, Javier NEGRO ${ }^{\ddagger}$ and Mariano A. DEL OLMO $\ddagger$ \\ † Departamento de Matemática Aplicada, Universidad de Valladolid, E-47011, Valladolid, Spain \\ E-mail: juacal@eis.uva.es \\ ¥Departamento de Física Teórica, Universidad de Valladolid, E-47011, Valladolid, Spain \\ E-mail: jnegro@fta.uva.es,olmo@fta.uva.es
}

Received November 14, 2008, in final form March 18, 2009; Published online April 01, 2009

doi:10.3842/SIGMA.2009.039

\begin{abstract}
We present an algebraic study of a kind of quantum systems belonging to a family of superintegrable Hamiltonian systems in terms of shape-invariant intertwinig operators, that span pairs of Lie algebras like $(s u(n), s o(2 n))$ or $(s u(p, q), s o(2 p, 2 q))$. The eigenstates of the associated Hamiltonian hierarchies belong to unitary representations of these algebras. It is shown that these intertwining operators, related with separable coordinates for the system, are very useful to determine eigenvalues and eigenfunctions of the Hamiltonians in the hierarchy. An study of the corresponding superintegrable classical systems is also included for the sake of completness.
\end{abstract}

Key words: superintegrable systems; intertwining operators; dynamical algebras

2000 Mathematics Subject Classification: 17B80; 81R12; 81R15

\section{Introduction}

It is well known that a Hamiltonian system (HS) in a configuration space of dimension $n$, is said to be integrable if there are $n$ constants of motion, including the Hamiltonian $H$, which are independent and in involution. If the systems has $0<k \leq n-1$ additional constants of motion then, it is called superintegrable. The physical system is said to be maximally superintegrable if there exist $2 n-1$ invariants well defined in phase-space. The superintegrable Hamiltonian systems (SHS) share nice properties. For instance, they admit separation of variables in more than one coordinate system for the Hamilton-Jacobi equation in the classical case and for the Schrödinger equation in the quantum case. Let us mention also that the finite classical trajectories are closed (periodic), while the discrete energy levels are degenerate in the quantum case.

There is a limited number of this kind of physical systems as can be found in the works by Evans [1]. More recently, we quote the deformed algebra approach to superintegrability by Daskaloyannis and collaborators $[2,3]$ and the superintegrability in constant curvature configuration spaces $[4,5,6]$. Among a long list of contributions we can also mention two former references. In 1975 Lakshmanan and Eswaran [7] analyzed the isotropic oscillator on a 3-sphere and in 1979 motived by this work Higgs [8] studied versions of the Coulomb potential and of the harmonic oscillator living in the $N$-dimensional sphere and having $S O(N+1)$ and $S U(N)$ symmetry in classical and in quantum mechanics, respectively.

\footnotetext{
${ }^{\star}$ This paper is a contribution to the Proceedings of the VIIth Workshop "Quantum Physics with NonHermitian Operators" (June 29 - July 11, 2008, Benasque, Spain). The full collection is available at http://www.emis.de/journals/SIGMA/PHHQP2008.html
} 
Some years ago a new family of SHS, was constructed from a group-theoretical method based on the symmetry reduction. These systems come, using the Marsden-Weinstein reduction [9], from free systems in $\mathbb{C}^{p, q}$ presenting an initial $U(p, q)$-symmetry [10]

$$
H=\frac{c}{4} g^{\bar{\mu} \nu} \bar{p}_{\mu} p_{\nu} \quad \stackrel{\mathrm{MW}}{\longrightarrow} \mathrm{reduction} \quad H^{r}=\frac{c}{4} g^{\mu \nu} p_{s^{\mu}} p_{s^{\nu}}+V(s)
$$

where the bar stands for the complex conjugate and $V(s)$ is a potential in terms of the real coordinates $\left(s^{\mu}\right)$. These SHS are living in configuration spaces of constant curvature $(S O(p, q)$ homogeneous spaces).

Although the quantum version of these systems is well known and can be exhaustively studied in all its aspects with standard procedures $[10,11,12,13,14]$, we present here a new perspective based on intertwining operators (IO), a form of Darboux transformations [15], that will allow us to study them from an algebraic point of view. The associated IO's close Lie algebras that take into account the symmetry properties of the systems and permit to describe these SHS in terms of representations of such "intertwining symmetry" algebras (or "dynamical algebras" [16]).

The intertwining operators are first order differential operators, $A$, connecting different Hamiltonians, $H, H^{\prime}$, in the same hierarchy, i.e., $A H=H^{\prime} A$. In the cases under study it is obtained a complete set of such IO's, in the sense that any of the Hamiltonians of the hierarchy can be expressed in terms of these operators. As we will see later, the IO's are associated to systems of separable coordinates for the Hamiltonians. The study of the IO's associated to integrable Hamiltonians has been made, for instance, in $[17,18,19]$ and, following this line of research, we will supply here other non-trivial applications by means of the above mentioned family of SHS. From the perspective of the IO's, we present a natural extension to higher dimensions of the intertwining (Darboux) transformations of the Schrödinger equation for one-dimensional quantum systems [20]. When a system of separable coordinates is used, any Hamiltonian of this family of SHS gives rise to a coupled set of $n$-differential equations, which can be factorized one by one.

In principle, we will present two particular SHS that we denote $u(3)$-system [21] and $u(2,1)$ system [22], but the generalization to higher $u(p, q)$-systems is evident. They are living in configuration spaces of constant curvature $(S O(3)$ and $S O(2,1)$-homogeneous spaces, respectively): a 2D sphere and a 2D hyperboloid of two-sheets. By extending well known methods in one-dimension to higher-dimensional systems we obtain a wide set of IO's closing the dynamical Lie algebras $u(3)$ or $u(2,1)$. These initial intertwining symmetry algebras can be enlarged by considering discrete symmetry operators obtaining, respectively, the $s o(6)$ and $s o(4,2)$ Lie algebras of IO's. This approach gives a simple explanation of the main features of these physical systems. For instance, it allows us to characterize the discrete spectrum and the corresponding eigenfunctions of the system by means of (finite/infinite) irreducible unitary representations (IUR) of the (compact/non-compact) intertwining symmetry algebras. We can compute the ground state and characterize the representation space of the wave-functions which share the same energy. The organization of the paper is as follows. In Section 2 we introduce the classical superintegrable Hamiltonian family under consideration. In Section 3 we focus on the quantum systems and show how to build the IO's connecting hierarchies of these kind of Hamiltonians. It is seen that these operators close a $s u(2,1)$ or a $s u(3)$ Lie algebra. The Hamiltonians are related to the second order Casimirs of such algebras, while the discrete spectrum of the Hamiltonians is related to their IUR's. Next, a broader class of IO's is defined leading to the $s o(4,2)$ or so(6) Lie algebras, and it is shown how this new structure helps us to understand better the Hamiltonians in the new hierarchies. Finally, some remarks and conclusions in Section 4 will end the paper. 


\section{Superintegrable $S U(p, q)$-Hamiltonian systems}

Let us consider the free Hamiltonian

$$
H=4 g^{\mu \bar{\nu}} p_{\mu} \bar{p}_{\nu}, \quad \mu, \nu=0, \ldots, n=p+q-1,
$$

(by $p_{\mu}$ we denote the conjugate momenta) defined in the configuration space

$$
\frac{S U(p, q)}{S U(p-1, q) \times U(1)}
$$

which is an Hermitian hyperbolic space with metric $g_{\mu \nu}$ and coordinates $y^{\mu} \in \mathbb{C}$ such that

$$
g_{\bar{\mu} \nu} \bar{y}^{\mu} y^{\nu}=1
$$

The geometry and properties of this kind of spaces are described in [23] and [12].

Using a maximal Abelian subalgebra (MASA) of $s u(p, q)[24]$ the reduction procedure allows us to obtain a reduced Hamiltonian, which is not free, lying in the corresponding reduced space, a homogeneous $S O(p, q)$-space $[10,11]$

$$
H=\frac{1}{2} g^{\mu \nu} p_{s^{\mu}} p_{s^{\nu}}+V(s)
$$

where $V(s)$ is a potential depending on the real coordinates $s^{\mu}$ satisfying $g_{\mu \nu} s^{\mu} s^{\nu}=1$.

The set of complex coordinates $y^{\mu}$ after the reduction procedure becomes a set of ignorable variables $x^{\mu}$ and the actual real coordinates $s^{\mu}$. A way to implement the symmetry reduction is as follows. Let $Y_{\mu}, \mu=0, \ldots, n$, be a basis of the considered MASA of $u(p, q)$ constituted only by pure imaginary matrices (this is a basic hypothesis in the reduction procedure). Then the relation between old $\left(y^{\mu}\right)$ and new coordinates $\left(x^{\mu}, s^{\mu}\right)$ is

$$
y^{\mu}=B(x)_{\nu}^{\mu} s^{\nu}, \quad B(x)=\exp \left(x^{\mu} Y_{\mu}\right)
$$

The fact that the $\left(x^{\mu}\right)$ are the parameters of the transformation associated to the MASA of $u(p, q)$ used in the reduction, assures the ignorability of the $x$ coordinates (in other words, the vector fields corresponding to the MASA are straightened out in these coordinates). The Jacobian matrix, $J$, corresponding to the coordinate transformation $((y, \bar{y}) \rightarrow(x, s))$ is given explicitly by

$$
J=\frac{\partial(y, \bar{y})}{\partial(x, s)}=\left(\begin{array}{cc}
A & B \\
\bar{A} & \bar{B}
\end{array}\right)
$$

where

$$
A_{\nu}^{\mu}=\frac{\partial y^{\mu}}{\partial x^{\nu}}=\left(Y_{\nu}\right)_{\rho}^{\mu} y^{\rho}
$$

The expression of the Hamiltonian (1) in the new coordinates $s$ is

$$
H=c\left(\frac{1}{2} g^{\mu \nu} p_{\mu} p_{\nu}+V(s)\right), \quad V(s)=p_{x}^{T}\left(A^{\dagger} K A\right)^{-1} p_{x}
$$

where $p_{x}$ are the constant momenta associated to the ignorable coordinates $x$ and $K$ is the matrix defined by the metric $g$. 


\subsection{A classical superintegrable $u(3)$-Hamiltonian}

To obtain the classical superintegrable Hamiltonian associated to $s u(3)$, using the reduction procedure sketched before, we proceed as follows: let us consider the basis of $s u(3)$ determined by $3 \times 3$ matrices $X_{1}, \ldots, X_{8}$, whose explicit form, using the metric $K=\operatorname{diag}(1,1,1)$, is

$$
\begin{aligned}
& X_{1}=\left(\begin{array}{ccc}
i & 0 & 0 \\
0 & -i & 0 \\
0 & 0 & 0
\end{array}\right), \quad X_{2}=\left(\begin{array}{ccc}
0 & 0 & 0 \\
0 & i & 0 \\
0 & 0 & -i
\end{array}\right), \quad X_{3}=\left(\begin{array}{ccc}
0 & 1 & 0 \\
-1 & 0 & 0 \\
0 & 0 & 0
\end{array}\right), \quad X_{4}=\left(\begin{array}{ccc}
0 & i & 0 \\
i & 0 & 0 \\
0 & 0 & 0
\end{array}\right), \\
& X_{5}=\left(\begin{array}{ccc}
0 & 0 & 1 \\
0 & 0 & 0 \\
-1 & 0 & 0
\end{array}\right), \quad X_{6}=\left(\begin{array}{ccc}
0 & 0 & i \\
0 & 0 & 0 \\
i & 0 & 0
\end{array}\right), \quad X_{7}=\left(\begin{array}{ccc}
0 & 0 & 0 \\
0 & 0 & 1 \\
0 & -1 & 0
\end{array}\right), \quad X_{8}=\left(\begin{array}{ccc}
0 & 0 & 0 \\
0 & 0 & i \\
0 & i & 0
\end{array}\right) \text {. }
\end{aligned}
$$

There is only one MASA for $s u(3)$ : the Cartan subalgebra, generated by the matrices

$$
\operatorname{diag}(i,-i, 0), \quad \operatorname{diag}(0, i,-i) .
$$

So, we can generate only one $s u(3)$-Hamiltonian system. In order to facilitate the computations we shall use the following basis for the corresponding MASA in $u(3)$

$$
Y_{0}=\operatorname{diag}(i, 0,0), \quad Y_{1}=\operatorname{diag}(0, i, 0), \quad Y_{2}=\operatorname{diag}(0,0, i) .
$$

The actual real coordinates $s$ are related to the complex coordinates $y$ by

$$
y_{\mu}=s_{\mu} e^{i x_{\mu}}, \quad \mu=0,1,2,
$$

and the Hamiltonian can be written as

$$
H=\frac{1}{2}\left(p_{0}^{2}+p_{1}^{2}+p_{2}^{2}\right)+V(s), \quad V(s)=\frac{m_{0}^{2}}{s_{0}^{2}}+\frac{m_{1}^{2}}{s_{1}^{2}}+\frac{m_{2}^{2}}{s_{2}^{2}},
$$

which lies in the 2-sphere $\left(s_{0}\right)^{2}+\left(s_{1}\right)^{2}+\left(s_{2}\right)^{2}=1$, with $m_{0}, m_{1}, m_{2} \in \mathbb{R}$. The system is superintegrable since there exist three invariants of motion

$$
R_{\mu \nu}=\left(s_{\mu} p_{\nu}-s_{\nu} p_{\mu}\right)^{2}+\left(m_{\mu} \frac{s_{\nu}}{s_{\mu}}+m_{\nu} \frac{s_{\mu}}{s_{\nu}}\right)^{2}, \quad \mu<\nu, \quad \mu=0,1, \quad \nu=1,2 .
$$

The constants of motion $R_{\mu \nu}$ can be written in terms of the basis of $s u(3)$ (in the realization as function of $s_{\mu}$ and $p_{\mu}$ )

$$
Q_{1} \equiv R_{01}=X_{3}^{2}+X_{4}^{2}, \quad Q_{2} \equiv R_{02}=X_{5}^{2}+X_{6}^{2}, \quad Q_{3} \equiv R_{12}=X_{7}^{2}+X_{8}^{2},
$$

and the sum of these invariants is the Hamiltonian (3) up to an additive constant

$$
H=Q_{1}+Q_{2}+Q_{3}+\text { cnt. }
$$

The quadratic Casimir of $s u(3)$ can be also written in terms of the constants of motion and the second order operators in the enveloping algebra of the compact Cartan subalgebra of su(3)

$$
\mathcal{C}_{s u(3)}=3 Q_{1}+3 Q_{2}+3 Q_{3}+4 X_{1}^{2}+2\left[X_{1}, X_{2}\right]_{+}+4 X_{2}^{2} .
$$

The Hamiltonian is in involution with all the three constants of motion, i.e. $\left[H, Q_{i}\right]=0, i=$ $1,2,3$. However, the $Q_{i}$ 's do not commute among them

$$
\begin{aligned}
{\left[Q_{1}, Q_{2}\right] } & =\left[Q_{3}, Q_{1}\right]=\left[Q_{2}, Q_{3}\right] \\
& =-\left[X_{3},\left[X_{5}, X_{7}\right]_{+}\right]_{+}-\left[X_{3},\left[X_{6}, X_{8}\right]_{+}\right]_{+}+\left[X_{4},\left[X_{5}, X_{8}\right]_{+}\right]_{+}-\left[X_{4},\left[X_{6}, X_{7}\right]_{+}\right]_{+} .
\end{aligned}
$$

So, the system (3) is superintegrable. 


\subsubsection{The Hamilton-Jacobi equation for the $u(3)$-system}

The solutions of the motion problem for this system can be obtained solving the corresponding Hamilton-Jacobi (HJ) equation in an appropriate coordinate system, such that the HJ equation separates into a system of ordinary differential equations.

The 2-sphere can be parametrized on spherical coordinates $\left(\phi_{1}, \phi_{2}\right)$ around the $s_{2}$ axis by

$$
s_{0}=\cos \phi_{2} \cos \phi_{1}, \quad s_{1}=\cos \phi_{2} \sin \phi_{1}, \quad s_{2}=\sin \phi_{2},
$$

where $\phi_{1} \in[0,2 \pi)$ and $\phi_{2} \in[\pi / 2,3 \pi / 2]$. Then, the Hamiltonian (3) is rewritten as

$$
H=\frac{1}{2}\left(p_{\phi_{2}}^{2}+\frac{p_{\phi_{1}}^{2}}{\cos ^{2} \phi_{2}}\right)+\frac{1}{\cos ^{2} \phi_{2}}\left(\frac{m_{0}^{2}}{\cos ^{2} \phi_{1}}+\frac{m_{1}^{2}}{\sin ^{2} \phi_{1}}\right)+\frac{m_{2}^{2}}{\sin ^{2} \phi_{2}} .
$$

The potential is periodic and has singularities along the coordinate lines $\phi_{1}=0, \pi / 2, \pi, 3 \pi / 2$ and $\phi_{2}=\pi / 2,3 \pi / 2$, and there is a unique minimum inside each domain of regularity.

The invariants $Q_{i}$ can be rewritten in spherical coordinates taking the explicit form

$$
\begin{aligned}
Q_{1}= & \frac{1}{2} p_{\phi_{1}}^{2}+\frac{m_{0}^{2}}{\cos } \phi_{1}+\frac{m_{1} 2^{2}}{\sin } \phi_{1}, \\
Q_{2}= & \tan ^{2} \phi_{2}\left(\frac{1}{2} p_{\phi_{1}}^{2} \sin ^{2} \phi_{1}+\frac{m_{0}^{2}}{\cos } \phi_{1}\right)+\cos ^{2} \phi_{1}\left(\frac{1}{2} p_{\phi_{2}}^{2}+\frac{m_{2}^{2}}{\tan } \phi_{2}\right) \\
& +\frac{1}{2} p_{\phi_{1}} p_{\phi_{2}} \sin 2 \phi_{1} \tan \phi_{2}, \\
Q_{3}= & \tan ^{2} \phi_{2}\left(\frac{1}{2} p_{\phi_{1}}^{2} \cos ^{2} \phi_{1}+\frac{m_{1}^{2}}{\sin } \phi_{1}\right)+\sin ^{2} \phi_{1}\left(\frac{1}{2} p_{\phi_{2}}^{2}+\frac{m_{2}^{2}}{\tan } \phi_{2}\right) \\
& -\frac{1}{2} p_{\phi_{1}} p_{\phi_{2}} \sin 2 \phi_{1} \tan \phi_{2} .
\end{aligned}
$$

Now, the HJ equation takes the form

$$
\frac{1}{2}\left(\frac{\partial S}{\partial \phi_{2}}\right)^{2}+\frac{m_{2}^{2}}{\sin ^{2} \phi_{2}}+\frac{1}{\cos ^{2} \phi_{2}}\left(\frac{1}{2}\left(\frac{\partial S}{\partial \phi_{1}}\right)^{2}+\frac{m_{0}^{2}}{\cos ^{2} \phi_{1}}+\frac{m_{1}^{2}}{\sin ^{2} \phi_{1}}\right)=E .
$$

It separates into two ordinary differential equations taking into account that the solution of the HJ equation can be written as $S\left(\phi_{1}, \phi_{2}\right)=S_{1}\left(\phi_{1}\right)+S_{2}\left(\phi_{2}\right)-E t$. Thus,

$$
\begin{aligned}
& \frac{1}{2}\left(\frac{\partial S_{1}}{\partial \phi_{1}}\right)^{2}+\frac{m_{0}^{2}}{\cos ^{2} \phi_{1}}+\frac{m_{1}^{2}}{\sin ^{2} \phi_{1}}=\alpha_{1}, \\
& \frac{1}{2}\left(\frac{\partial S_{2}}{\partial \phi_{2}}\right)^{2}+\frac{m_{2}^{2}}{\sin ^{2} \phi_{2}}+\frac{\alpha_{1}}{\cos ^{2} \phi_{2}}=\alpha_{2},
\end{aligned}
$$

where $\alpha_{2}=E$ and $\alpha_{1}$ are the separation constants (which are positive). Each one of these two equations is formaly similar to those of the corresponding one-dimensional problem [13]. The solutions of both HJ equations are easily computed and can be found as particular cases in [12]. Notice that all the orbits in a neighborhood of a critical point (center) are closed and, hence, the corresponding trajectories are periodic.

The explicit solutions, when we restrict us to the domain $0<\phi_{1}, \phi_{2}<\pi / 2$, are

$$
\cos ^{2} \phi_{2}=\frac{1}{2 E}\left[b_{2}+\sqrt{b_{2}^{2}-4 \alpha_{1} E} \cos 2 \sqrt{2 E} t\right],
$$




$$
\begin{aligned}
\cos ^{2} \phi_{1}= & \frac{1}{2 \alpha_{1}}\left[b_{1}+\frac{1}{\cos ^{2} \phi_{2}}\left[\frac{b_{1}^{2}-4 \alpha_{1} m_{0}^{2}}{b_{2}^{2}-4 \alpha_{1} E}\right]^{1 / 2}\left(\left(b_{2} \cos ^{2} \phi_{2}-2 \alpha_{1}\right) \sin 2 \sqrt{2 \alpha_{1}} \beta_{1}\right.\right. \\
& \left.\left.+2 \sqrt{\alpha_{1}}\left[\left(b_{2}-E \cos ^{2} \phi_{2}\right) \cos ^{2} \phi_{2}-\alpha_{1}\right]^{1 / 2} \cos 2 \sqrt{2 \alpha_{1}} \beta_{1}\right)\right]
\end{aligned}
$$

where $b_{1}=\alpha_{1}+m_{0}^{2}-m_{1}^{2}$ and $b_{2}=E+\alpha_{1}-m_{2}^{2}$. Inside the domain the minimum for the potential is at the point $\left(\phi_{1}=\arctan \sqrt{m_{1} / m_{0}}, \phi_{2}=\arctan \sqrt{\left.m_{2} /\left(m_{0}+m_{1}\right)\right)}\right.$, and its value is $V_{\min }=\left(m_{0}+m_{1}+m_{2}\right)^{2}$. Hence, the energy $E$ is bounded from below $E \geq\left(m_{0}+m_{1}+m_{2}\right)^{2}$.

\subsection{A classical superintegrable $u(2,1)$-Hamiltonian}

In a similar way to the preceding case $u(3)$ of Section 2.1 , it is enough to find an appropriate basis of $u(2,1)$, for instance

$$
\begin{aligned}
X_{1}=\left(\begin{array}{ccc}
i & 0 & 0 \\
0 & -i & 0 \\
0 & 0 & 0
\end{array}\right), & X_{2}=\left(\begin{array}{ccc}
0 & 0 & 0 \\
0 & i & 0 \\
0 & 0 & -i
\end{array}\right), & X_{3}=\left(\begin{array}{ccc}
0 & 1 & 0 \\
-1 & 0 & 0 \\
0 & 0 & 0
\end{array}\right), & X_{4}=\left(\begin{array}{ccc}
0 & i & 0 \\
i & 0 & 0 \\
0 & 0 & 0
\end{array}\right), \\
X_{5}=\left(\begin{array}{lll}
0 & 0 & 1 \\
0 & 0 & 0 \\
1 & 0 & 0
\end{array}\right), & X_{6}=\left(\begin{array}{ccc}
0 & 0 & i \\
0 & 0 & 0 \\
-i & 0 & 0
\end{array}\right), & X_{7}=\left(\begin{array}{ccc}
0 & 0 & 0 \\
0 & 0 & 1 \\
0 & 1 & 0
\end{array}\right), & X_{8}=\left(\begin{array}{ccc}
0 & 0 & 0 \\
0 & 0 & i \\
0 & -i & 0
\end{array}\right),
\end{aligned}
$$

and to follow the same procedure. However, the Lie algebra $s u(2,1)$ has four MASAS [24]: the compact Cartan subalgebra like $u(3)$, the noncompact Cartan subalgebra, the orthogonally decomposable subalgebra and the nilpotent subalgebra. For our purposes in this work we will only consider the symmetry reduction by the compact Cartan subalgebra, although we could generate other three SHS with the remaining MASAs. It is also possible to use the same matrices of $u(3)$ (2) to build up a basis of the compact Cartan subalgebra.

We find the following reduced Hamiltonian

$$
H=\frac{1}{2} c\left(-p_{0}^{2}-p_{1}^{2}+p_{2}^{2}\right)+\frac{m_{0}^{2}}{s_{0}^{2}}+\frac{m_{1}^{2}}{s_{1}^{2}}-\frac{m_{2}^{2}}{s_{2}^{2}},
$$

lying in the 2-dimensional two-sheet hyperboloid $-s_{0}^{2}-s_{1}^{2}+s_{2}^{2}=1$ and with $c$ a constant. The potential constants, $m_{i}$, can be chosen non-negative real numbers.

Parametrizing the two-sheet hyperboloid by using an 'analogue' of the spherical coordinates

$$
s_{0}=\sinh \xi \cos \theta, \quad s_{1}=\sinh \xi \sin \theta, \quad s_{2}=\cosh \xi,
$$

with $0 \leq \theta<\pi / 2$ and $0 \leq \xi<\infty$, the Hamiltonian can be rewritten if $c=-1$ as

$$
H=\frac{1}{2}\left(p_{\xi}^{2}+\frac{p_{\theta}^{2}}{\sinh ^{2} \xi}\right)+\frac{1}{\sinh ^{2} \xi}\left(\frac{m_{0}^{2}}{\cos ^{2} \theta}+\frac{m_{1}^{2}}{\sin ^{2} \theta}\right)-\frac{m_{2}^{2}}{\cosh ^{2} \xi} .
$$

The potential is regular inside the domain of the variables and there is a saddle point for the values $\theta=\arctan \sqrt{m_{1} / m_{0}}$ and $\left.\xi=\arg \tanh \sqrt{m_{2}\left(m_{0}+m_{1}\right)}\right)$ if $m_{0}+m_{1}>m_{2}$.

The quadratic constants of motion in terms of the enveloping algebra of $s u(2,1)$ are

$$
Q_{1}=X_{3}^{2}+X_{4}^{2}, \quad Q_{2}=X_{5}^{2}+X_{6}^{2}, \quad Q_{3}=X_{7}^{2}+X_{8}^{2},
$$

and the sum of these invariants gives also the Hamiltonian up to an additive constant

$$
H=-Q_{1}+Q_{2}+Q_{3}+\text { cnt }
$$


The quadratic Casimir of $s u(2,1)$ is

$$
\mathcal{C}_{s u(2,1)}=3 Q_{1}-3 Q_{2}-3 Q_{3}+4 X_{1}^{2}+2\left[X_{1}, X_{2}\right]_{+}+4 X_{2}^{2} .
$$

The Hamiltonian is in involution with all the three constants of motion that do not commute among themselves

$$
\begin{aligned}
{\left[Q_{1}, Q_{2}\right] } & =\left[Q_{3}, Q_{1}\right]=\left[Q_{3}, Q_{2}\right] \\
& =-\left[X_{3},\left[X_{5}, X_{7}\right]_{+}\right]_{+}-\left[X_{3},\left[X_{6}, X_{8}\right]_{+}\right]_{+}+\left[X_{4},\left[X_{5}, X_{8}\right]_{+}\right]_{+}-\left[X_{4},\left[X_{6}, X_{7}\right]_{+}\right]_{+} .
\end{aligned}
$$

The explicit form of the invariants of motion in terms of the coordinates $\xi$ and $\theta$ is

$$
\begin{aligned}
& Q_{1}=\frac{1}{2} p_{\theta}^{2}+\frac{m_{0}^{2}}{\cos ^{2} \theta}+\frac{m_{1}^{2}}{\sin ^{2} \theta}, \\
& Q_{2}=\operatorname{coth}^{2} \xi\left(\frac{1}{2} p_{\theta}^{2} \sin ^{2} \theta+\frac{m_{0}^{2}}{\cos ^{2} \theta}\right)+\cos ^{2} \theta\left(\frac{1}{2} p_{\xi}^{2}+\frac{m_{2}^{2}}{\operatorname{coth}^{2} \xi}\right)+\frac{1}{2} p_{\theta} p_{\xi} \sin 2 \theta \operatorname{coth} \xi \\
& Q_{3}=\operatorname{coth}^{2} \xi\left(\frac{1}{2} p_{\theta}^{2} \cos ^{2} \theta+\frac{m_{1}^{2}}{\sin ^{2} \theta}\right)+\sin ^{2} \theta\left(\frac{1}{2} p_{\xi}^{2}+\frac{m_{2}^{2}}{\operatorname{coth}^{2} \xi}\right)-\frac{1}{2} p_{\theta} p_{\xi} \sin 2 \theta \operatorname{coth} \xi
\end{aligned}
$$

\section{Superintegrable quantum systems}

In the previous Section 2 we have described some classical superintegrable systems. Now we will study their quantum versions. In order to construct the quantum version of both systems, let us proceed in the following way. By inspection of the classical Hamiltonians (3) and (4) and their constants of motions we can relate the terms like $s_{\mu} p_{\nu} \pm s_{\nu} p_{\mu}$ with generators of "rotations" when $p_{\mu} \rightarrow \partial_{\mu}$ in the plane $X_{\mu} X_{\nu}$. Moreover, since the Hamiltonian is the sum of the three constants of motion up to constants we can write a quantum Hamiltonian as a linear combination of $J_{0}^{2}$, $J_{1}^{2}$ and $J_{2}^{2}$, being $J_{0}, J_{1}$ and $J_{2}$ the infinitesimal generators of "rotations" in the plane $X_{1} X_{2}$, $X_{0} X_{2}$ and $X_{0} X_{1}$ around the axis $X_{0}, X_{1}$ and $X_{2}$, respectively. According to the signature of the metric the rotations will be compact or noncompact, so, the generators will span so(3) or $s o(2,1)$ and for our purposes we will take a differential realization of them. In other words, the Casimir operator of $s o(3)\left(C_{s o(3)}=J_{0}^{2}+J_{1}^{2}+J_{2}^{2}\right)$ or of $s o(2,1)\left(C_{s o(2,1)}=J_{0}^{2}+J_{1}^{2}-J_{2}^{2}\right)$ gives the "kinetic" part of the corresponding Hamiltonian. In the following we will present a detailed study of the case related with $s u(2,1)$. The $s u(3)$-case is simply sketched and the interested reader can find more details in [21].

\subsection{Superintegrable quantum $u(2,1)$-system}

Let us consider the Hamiltonian

$$
H_{\ell}=-J_{0}^{2}-J_{1}^{2}+J_{2}^{2}+\frac{l_{0}^{2}-\frac{1}{4}}{s_{0}^{2}}+\frac{l_{1}^{2}-\frac{1}{4}}{s_{1}^{2}}-\frac{l_{2}^{2}-\frac{1}{4}}{s_{2}^{2}},
$$

which configuration space is the 2 -dimensional two-sheet hyperboloid $-s_{0}^{2}-s_{1}^{2}+s_{2}^{2}=1$, with $\ell=\left(l_{0}, l_{1}, l_{2}\right) \in \mathbb{R}^{3}$ (and $2 m=1$ ). The differential operators

$$
J_{0}=s_{1} \partial_{2}+s_{2} \partial_{1}, \quad J_{1}=s_{2} \partial_{0}+s_{0} \partial_{2}, \quad J_{2}=s_{0} \partial_{1}-s_{1} \partial_{0},
$$

constitute a realization of $s o(2,1)$ with Lie commutators

$$
\left[J_{0}, J_{1}\right]=-J_{2}, \quad\left[J_{2}, J_{0}\right]=J_{1}, \quad\left[J_{1}, J_{2}\right]=J_{0} .
$$


Using coordinates (5) the explicit expressions of the infinitesimal generators are

$$
J_{0}=\sin \theta \partial_{\xi}+\cos \theta \operatorname{coth} \xi \partial_{\theta}, \quad J_{1}=\cos \theta \partial_{\xi}-\sin \theta \operatorname{coth} \xi \partial_{\theta}, \quad J_{2}=\partial_{\theta} .
$$

They are anti-Hermitian operators inside the space of square-integrable functions with invariant measure $d \mu(\theta, \xi)=\sinh \xi d \theta d \xi$.

In these coordinates the Hamiltonian $H_{\ell}(6)$ has the expression

$$
H_{\ell}=-\partial_{\xi}^{2}-\operatorname{coth} \xi \partial_{\xi}-\frac{l_{2}^{2}-\frac{1}{4}}{\cosh ^{2} \xi}+\frac{1}{\sinh ^{2} \xi}\left[-\partial_{\theta}^{2}+\frac{l_{1}^{2}-\frac{1}{4}}{\sin ^{2} \theta}+\frac{l_{0}^{2}-\frac{1}{4}}{\cos ^{2} \theta}\right] \text {. }
$$

It can be separated in the variables $\xi$ and $\theta$, by choosing its eigenfunctions $\Phi_{\ell}$ in the form $\Phi_{\ell}(\theta, \xi)=f(\theta) g(\xi)$, obtaining a pair of separated equations

$$
\begin{aligned}
& H_{l_{0}, l_{1}}^{\theta} f(\theta) \equiv\left[-\partial_{\theta}^{2}+\frac{l_{1}^{2}-\frac{1}{4}}{\sin ^{2} \theta}+\frac{l_{0}^{2}-\frac{1}{4}}{\cos ^{2} \theta}\right] f(\theta)=\alpha f(\theta), \\
& {\left[-\partial_{\xi}^{2}-\operatorname{coth} \xi \partial_{\xi}-\frac{l_{2}^{2}-\frac{1}{4}}{\cosh ^{2} \xi}+\frac{\alpha}{\sinh ^{2} \xi}\right] g(\xi)=E g(\xi),}
\end{aligned}
$$

where $\alpha>0$ is a separation constant.

\subsubsection{A complete set of intertwining operators for $\boldsymbol{H}_{\ell}$}

The one-dimensional Hamiltonian $H_{l_{0}, l_{1}}^{\theta}(7)$ can be factorized as a product of first order operators $A^{ \pm}$and a constant $\lambda_{\left(l_{0}, l_{1}\right)}$

$$
\begin{aligned}
& H_{\left(l_{0}, l_{1}\right)}^{\theta}=A_{\left(l_{0}, l_{1}\right)}^{+} A_{\left(l_{0}, l_{1}\right)}^{-}+\lambda_{\left(l_{0}, l_{1}\right)}, \\
& A_{l_{0}, l_{1}}^{ \pm}= \pm \partial_{\theta}-\left(l_{0}+1 / 2\right) \tan \theta+\left(l_{1}+1 / 2\right) \cot \theta, \\
& \lambda_{\left(l_{0}, l_{1}\right)}=\left(l_{0}+l_{1}+1\right)^{2} .
\end{aligned}
$$

The fundamental relation between contiguous couples of operators $A^{ \pm}$,

$$
H_{\left(l_{0}, l_{1}\right)}^{\theta}=A_{\left(l_{0}, l_{1}\right)}^{+} A_{\left(l_{0}, l_{1}\right)}^{-}+\lambda_{\left(l_{0}, l_{1}\right)}=A_{\left(l_{0}+1, l_{1}+1\right)}^{-} A_{\left(l_{0}+1, l_{1}+1\right)}^{+}+\lambda_{\left(l_{0}+1, l_{1}+1\right)},
$$

allows us to construct a hierarchy of Hamiltonians

$$
\ldots, H_{l_{0}-1, l_{1}-1}^{\theta}, H_{l_{0}, l_{1}}^{\theta}, H_{l_{0}+1, l_{1}+1}^{\theta}, \ldots, H_{l_{0}+n, l_{1}+n}^{\theta}, \ldots
$$

which satisfy the recurrence relations

$$
A_{\left(l_{0}, l_{1}\right)}^{-} H_{\left(l_{0}, l_{1}\right)}^{\theta}=H_{\left(l_{0}+1, l_{1}+1\right)}^{\theta} A_{\left(l_{0}, l_{1}\right)}^{-}, \quad A_{\left(l_{0}, l_{1}\right)}^{+} H_{\left(l_{0}+1, l_{1}+1\right)}^{\theta}=H_{\left(l_{0}, l_{1}\right)}^{\theta} A_{\left(l_{0}, l_{1}\right)}^{+} .
$$

From the above relations we see that the operators $A_{\left(l_{0}, l_{1}\right)}^{ \pm}$act as shape invariant intertwining operators and also that $A_{\left(l_{0}, l_{1}\right)}^{-}$transforms eigenfunctions of $H_{\left(l_{0}, l_{1}\right)}^{\theta}$ into eigenfunctions of $H_{\left(l_{0}+1, l_{1}+1\right)}^{\theta}$, and viceversa for $A_{\left(l_{0}, l_{1}\right)}^{+}$, in such a way that the original and the transformed eigenfunctions have the same eigenvalue.

Hence, once the initial values for $\left(l_{0}, l_{1}\right)$ have been fixed we can build up an infinite set of Hamiltonians $\left\{H_{\left(l_{0}+n, l_{1}+n\right)}^{\theta}\right\}_{n \in \mathbb{Z}}$ connected by the set of operators $\left\{A_{\left(l_{0}+n, l_{1}+n\right)}^{ \pm}\right\}_{n \in \mathbb{Z}}$ (Hamiltonian 'hierarchy'). 


\subsubsection{The $u(2)$ 'dynamical' algebra}

We can define free-index operators $\hat{H}^{\theta}, \hat{A}^{ \pm}, \hat{A}$ starting from the set of index-depending operators

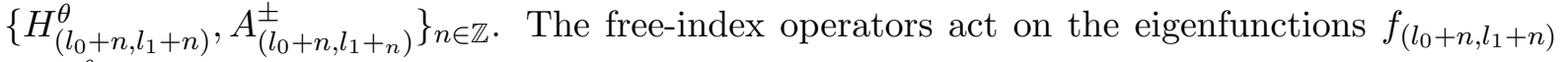
of $H_{\left(l_{0}+n, l_{1}+n\right)}^{\theta}$ as follows:

$$
\begin{aligned}
& \hat{H}^{\theta} f_{\left(l_{0}, l_{1}\right)}:=H_{\left(l_{0}, l_{1}\right)}^{\theta} f_{\left(l_{0}, l_{1}\right)}, \\
& \hat{A}^{-} f_{\left(l_{0}, l_{1}\right)}:=\frac{1}{2} A_{\left(l_{0}, l_{1}\right)}^{-} f_{\left(l_{0}, l_{1}\right)}, \\
& \hat{A}^{+} f_{\left(l_{0}+1, l_{1}+1\right)}:=\frac{1}{2} A_{\left(l_{0}, l_{1}\right)}^{+} f_{\left(l_{0}+1, l_{1}+1\right)}, \\
& \hat{A} f_{\left(l_{0}, l_{1}\right)}:=-\frac{1}{2}\left(l_{0}+l_{1}\right) f_{\left(l_{0}, l_{1}\right)} .
\end{aligned}
$$

With this convention the free-index operators close a $s u(2)$-algebra with commutators

$$
\left[\hat{A}, \hat{A}^{ \pm}\right]= \pm A^{ \pm}, \quad\left[\hat{A}^{+}, \hat{A}^{-}\right]=2 \hat{A}
$$

and including the operator $D f_{\left(l_{0}, l_{1}\right)}:=\left(l_{0}-l_{1}\right) f_{\left(l_{0}, l_{1}\right)}$, that commutes with the other three ones, we obtain a $u(2)$-algebra.

The fundamental states of some distinguished Hamiltonians are in relation with the IUR's of $s u(2)$. Thus, an eigenstate $f_{\left(l_{0}+n, l_{1}+n\right)}^{0}$ of $H_{\left(l_{0}+n, l_{1}+n\right)}^{\theta}$ will be a fundamental (highest or lowest weight) vector if

$$
A^{-} f_{\left(l_{0}+n, l_{1}+n\right)}^{0}=A_{\left(l_{0}+n, l_{1}+n\right)}^{-} f_{\left(l_{0}+n, l_{1}+n\right)}^{0}=0 .
$$

The solution of this equation,

$$
f_{\left(l_{0}+n, l_{1}+n\right)}^{0}\left(\theta_{1}\right)=N \cos ^{l_{0}+1 / 2+n}\left(\theta_{1}\right) \sin ^{l_{1}+1 / 2+n}\left(\theta_{1}\right)
$$

with $N$ a normalization constant and eigenvalue

$$
E_{\left(l_{0}+n, l_{1}+n\right)}^{0}=\lambda_{\left(l_{0}+n, l_{1}+n\right)}=\left(l_{0}+l_{1}+1+2 n\right)^{2},
$$

is also eigenfunction of $A$

$$
A f_{\left(l_{0}+n, l_{1}+n\right)}^{0}=A_{\left(l_{0}+n, l_{1}+n\right)} f_{\left(l_{0}+n, l_{1}+n\right)}^{0}=-\frac{1}{2}\left(l_{0}+l_{1}+2 n\right) f_{\left(l_{0}+n, l_{1}+n\right)}^{0} .
$$

The functions (10) are regular and square-integrable when $l_{0}, l_{1} \geq-1 / 2$. From (11) we can make the identification

$$
f_{\left(l_{0}+n, l_{1}+n\right)}^{0} \simeq\left|j_{n},-j_{n}\right\rangle
$$

with $j_{n}=\frac{1}{2}\left(l_{0}+l_{1}+2 n\right), n=0,1,2, \ldots$ The representation, $D^{j_{n}}$, fixed by $f_{\left(l_{0}+n, l_{1}+n\right)}^{0}$ will be a IUR of $s u(2)$ of dimension $2 j_{n}+1=l_{0}+l_{1}+2 n+1$ if $l_{0}+l_{1} \in \mathbb{Z}^{+}, n \in \mathbb{Z}^{+}$. The Hamiltonian $H^{\theta}$ can be written in terms of the Casimir of $s u(2), \mathcal{C}=A^{+} A^{-}+A(A-1)$, as follows

$$
H^{\theta}=4(\mathcal{C}+1 / 4)
$$

The other eigenstates in the representation $D^{j_{n}}$ are obtained applying recursively $A^{+}$. Thus,

$$
f_{\left(l_{0}, l_{1}\right)}^{n}=\left(A^{+}\right)^{n} f_{\left(l_{0}+n, l_{1}+n\right)}^{0}=A_{\left(l_{0}, l_{1}\right)}^{+} A_{\left(l_{0}+1, l_{1}+1\right)}^{+} \cdots A_{\left(l_{0}+n-1, l_{1}+n-1\right)}^{+} f_{\left(l_{0}+n, l_{1}+n\right)}^{0},
$$


and

$$
f_{\left(l_{0}, l_{1}\right)}^{n} \simeq\left|j_{n},-j_{n}+n\right\rangle .
$$

The explicit form of $f_{\left(l_{0}, l_{1}\right)}^{n}$ is

$$
f_{\left(l_{0}, l_{1}\right)}^{n}=\sin ^{l_{1}+1 / 2}\left(\phi_{1}\right) \cos ^{l_{0}+1 / 2}\left(\phi_{1}\right) P_{m}^{\left(l_{1}, l_{0}\right)}\left[\cos \left(2 \phi_{1}\right)\right],
$$

being $P_{m}$ the Jacobi polynomials, with eigenvalue

$$
E_{\left(l_{0}, l_{1}\right)}^{n}=\left(l_{0}+l_{1}+1+2 n\right)^{2}, \quad n \in \mathbb{Z}^{+} .
$$

Therefore, the eigenstates of the hierarchy $\left\{H_{\left(l_{0}+n, l_{1}+n\right)}\right\}_{n \in \mathbb{Z}}$ when $l_{0}+l_{1} \in \mathbb{Z}^{+}$can be 'organized' in IUR's of $s u(2)$ (or of $u(2)$ ). Notice that different fundamental states with values of $l_{0}$ and $l_{1}$, such that $j_{0}=\left(l_{0}+l_{1}\right) / 2$ is fixed, would lead to the same $j$-IUR of $s u(2)$, but different $u(2)$ IUR's may correspond to states with the same energy (because $\left.D f_{\left(l_{0}, l_{1}\right)}=\left(l_{0}-l_{1}\right) f_{\left(l_{0}, l_{1}\right)}\right)$. Hence, these results push us to find a larger algebra of operators such that all the eigenstates with the same energy belong to only one of its IUR's.

Since the IO's $A_{l_{0}, l_{1}}^{ \pm}$depend only on the $\theta$-variable, they can act also as IO's of the complete Hamiltonians $H_{\ell}(6)$ and its global eigenfunctions $\Phi_{\ell}$, leaving the parameter $l_{2}$ unchanged

$$
A_{\ell^{\prime}}^{-} H_{\ell^{\prime}}=H_{\ell} A_{\ell^{\prime}}^{-}, \quad A_{\ell^{\prime}}^{+} H_{\ell}=H_{\ell^{\prime}} A_{\ell^{\prime}}^{+},
$$

where $\ell=\left(l_{0}, l_{1}, l_{2}\right)$ and $\ell^{\prime}=\left(l_{0}-1, l_{1}-1, l_{2}\right)$. In this sense, many of the above relations can be straightforwardly extended under this global point of view.

\subsubsection{Second set of pseudo-spherical coordinates}

A second coordinate set, obtained from the noncompact rotations around the axes $s_{2}$ and $s_{0}$ respectively, and that allows us to parametrize the hyperboloid and separate the Hamiltonian is the following one

$$
s_{0}=\cosh \psi \sinh \chi, \quad s_{1}=\sinh \psi, \quad s_{2}=\cosh \psi \cosh \chi,
$$

with $-\infty<\psi<+\infty$ and $0 \leq \chi<+\infty$. In these coordinates the $s o(2,1)$-generators take the expressions

$$
J_{0}=-\tanh \psi \sinh \chi \partial_{\chi}+\cosh \chi \partial_{\psi}, \quad J_{1}=\partial_{\chi}, \quad J_{2}=\sinh \chi \partial_{\psi}-\tanh \psi \cosh \chi \partial_{\chi} .
$$

The explicit expression of the Hamiltonian is now

$$
H_{\ell}=-\partial_{\psi}^{2}-\tanh \psi \partial_{\psi}+\frac{l_{1}^{2}-\frac{1}{4}}{\sinh ^{2} \psi}+\frac{1}{\cosh ^{2} \psi}\left[-\partial_{\chi}^{2}+\frac{l_{0}^{2}-\frac{1}{4}}{\sinh ^{2} \chi}-\frac{l_{2}^{2}-\frac{1}{4}}{\cosh ^{2} \chi}\right] .
$$

It can be separated in the variables $\psi$ and $\chi$ considering the eigenfunctions $H_{\ell}$ of the form $\Phi(\chi, \psi)=f(\chi) g(\psi)$. Hence, we obtain the following two equations

$$
\begin{aligned}
& H_{l_{0}, l_{2}}^{\chi} f(\chi) \equiv\left[-\partial_{\chi}^{2}+\frac{l_{0}^{2}-\frac{1}{4}}{\sinh ^{2} \chi}-\frac{l_{2}^{2}-\frac{1}{4}}{\cosh ^{2} \chi}\right] f(\chi)=\alpha f(\chi), \\
& {\left[-\partial_{\psi}^{2}-\tanh \psi \partial_{\psi}+\frac{l_{1}^{2}-\frac{1}{4}}{\sinh ^{2} \psi}+\frac{\alpha}{\cosh ^{2} \psi}\right] g(\psi)=E g(\psi),}
\end{aligned}
$$

with $\alpha$ a separation constant. 
The Hamiltonian $H_{l_{0}, l_{2}}^{\chi}(12)$ can be factorized as a product of first order operators $B^{ \pm}$

$$
H_{l_{0}, l_{2}}^{\chi}=B_{l_{0}, l_{2}}^{+} B_{l_{0}, l_{2}}^{-}+\lambda_{l_{0}, l_{2}}=B_{l_{0}-1, l_{2}-1}^{-} B_{l_{0}-1, l_{2}-1}^{+}+\lambda_{l_{0}-1, l_{2}-1}
$$

being

$$
B_{l_{0}, l_{2}}^{ \pm}= \pm \partial_{\chi}+\left(l_{2}+1 / 2\right) \tanh \chi+\left(l_{0}+1 / 2\right) \operatorname{coth} \chi, \quad \lambda_{l_{0}, l_{2}}=-\left(1+l_{0}+l_{2}\right)^{2} .
$$

In this case the intertwining relations take the form

$$
B_{l_{0}-1, l_{2}-1}^{-} H_{l_{0}-1, l_{2}-1}^{\chi}=H_{l_{0}, l_{2}}^{\chi} B_{l_{0}-1, l_{2}-1}^{-}, \quad B_{l_{0}-1, l_{2}-1}^{+} H_{l_{0}, l_{2}}^{\chi}=H_{l_{0}-1, l_{2}-1}^{\chi} B_{l_{0}-1, l_{2}-1}^{+} .
$$

Hence, the operators $B^{ \pm}$connect eigenfunctions of $H_{l_{0}, l_{2}}^{\chi}$ in the following way

$$
B_{l_{0}-1, l_{2}-1}^{-}: f_{l_{0}-1, l_{2}-1} \rightarrow f_{l_{0}, l_{2}}, \quad B_{l_{0}-1, l_{2}-1}^{+}: f_{l_{0}, l_{2}} \rightarrow f_{l_{0}-1, l_{2}-1} .
$$

The operators $B_{l_{0}, l_{2}}^{ \pm}$can be also expressed in terms of $\xi$ and $\theta$

$$
B_{l_{0}, l_{2}}^{ \pm}= \pm\left(\cos \theta \partial_{\xi}-\sin \theta \operatorname{coth} \xi \partial_{\theta}\right)+\left(l_{2}+1 / 2\right) \tanh \xi \cos \theta+\left(l_{0}+1 / 2\right) \operatorname{coth} \xi \sec \theta .
$$

We also define new free-index operators

$$
\hat{B}^{-} f_{l_{0}, l_{2}}:=\frac{1}{2} B_{l_{0}, l_{2}}^{-} f_{l_{0}, l_{2}}, \quad \hat{B}^{+} f_{l_{0}, l_{2}}:=\frac{1}{2} B_{l_{0}, l_{2}}^{+} f_{l_{0}, l_{2}}, \quad \hat{B} f_{l_{0}, l_{2}}:=-\frac{1}{2}\left(l_{0}+l_{2}\right) f_{l_{0}, l_{2}},
$$

that close a $s u(1,1)$ Lie algebra

$$
\left[\hat{B}^{+}, \hat{B}^{-}\right]=-2 \hat{B}, \quad\left[\hat{B}, \hat{B}^{ \pm}\right]= \pm \hat{B}^{ \pm}
$$

Since $s u(1,1)$ is non-compact, its IUR's are infinite-dimensional. In this case we are interested in the discrete series having a fundamental state annihilated by the lowering operator

$$
B^{-} f_{l_{0}, l_{2}}^{0}=0
$$

The explicit expression of these states is

$$
f_{l_{0}, l_{2}}^{0}(\chi)=N(\cosh \chi)^{l_{2}+1 / 2}(\sinh \chi)^{l_{0}+1 / 2},
$$

where $N$ is a normalization constant. In order to have a regular and square-integrable function we impose that

$$
l_{0} \geq-1 / 2, \quad-k_{1} \equiv l_{0}+l_{2}<-1 \text {. }
$$

Since $\hat{B} f_{l_{0}, l_{2}}^{0}=-\frac{1}{2}\left(l_{0}+l_{2}\right) f_{l_{0}, l_{2}}^{0}$, the lowest weight of this infinite-dimensional IUR of $s u(1,1)$ is characterized by

$$
j_{1}^{\prime}=k_{1} / 2>1 / 2 .
$$

The IO's $\hat{B}^{ \pm}$can also be considered as intertwining operators of the Hamiltonians $H_{\ell}$ linking their eigenfunctions $\Phi_{\ell}$, similarly to the IO's $\hat{A}^{ \pm}$, described before, but now with $l_{1}$ remaining unchanged. 


\subsubsection{Third set of pseudo-spherical coordinates}

A third set of coordinates is obtained from the noncompact rotations around the axes $s_{1}$ and $s_{0}$, respectively. It gives rise to the following parametrization of the hyperboloid

$$
s_{0}=\sinh \phi, \quad s_{1}=\cosh \phi \sinh \beta, \quad s_{2}=\cosh \phi \cosh \beta,
$$

with $0 \leq \phi<+\infty$ and $-\infty<\beta<+\infty$. The infinitesimal generators have the expressions

$$
J_{0}=\partial_{\beta}, \quad J_{1}=\cosh \beta \partial_{\phi}-\tanh \phi \sinh \beta \partial_{\beta}, \quad J_{2}=-\sinh \beta \partial_{\phi}+\tanh \phi \cosh \beta \partial_{\beta} .
$$

Hence, the Hamiltonian now takes the form

$$
H_{\ell}=-\partial_{\phi}^{2}-\tanh \phi \partial_{\phi}+\frac{l_{0}^{2}-\frac{1}{4}}{\sinh ^{2} \phi}+\frac{1}{\cosh ^{2} \phi}\left[-\partial_{\beta}^{2}+\frac{l_{1}^{2}-\frac{1}{4}}{\sinh ^{2} \beta}-\frac{l_{2}^{2}-\frac{1}{4}}{\cosh ^{2} \beta}\right],
$$

and it separates in the variables $\phi, \beta$ in terms of its eigenfunctions $\Phi(\beta, \phi)=f(\beta) g(\phi)$

$$
\begin{aligned}
& H_{l_{1}, l_{2}}^{\beta} f(\beta) \equiv\left[-\partial_{\beta}^{2}+\frac{l_{1}^{2}-\frac{1}{4}}{\sinh ^{2} \beta}-\frac{l_{2}^{2}-\frac{1}{4}}{\cosh ^{2} \beta}\right] f(\beta)=\alpha f(\beta), \\
& {\left[-\partial_{\phi}^{2}-\tanh \phi \partial_{\phi}+\frac{l_{0}^{2}-\frac{1}{4}}{\sinh ^{2} \phi}+\frac{\alpha}{\cosh ^{2} \phi}\right] g(\phi)=E g(\phi),}
\end{aligned}
$$

with the separation constant $\alpha$.

The second order operator $H_{l_{1}, l_{2}}^{\beta}$ can be factorized as a product of first order operators $C^{ \pm}$

$$
H_{l_{1}, l_{2}}^{\beta}=C_{l_{1}, l_{2}}^{+} C_{l_{1}, l_{2}}^{-}+\lambda_{l_{1}, l_{2}}=C_{l_{1}+1, l_{2}-1}^{-} C_{l_{1}+1, l_{2}-1}^{+}+\lambda_{l_{1}+1, l_{2}-1}
$$

being

$$
C_{l_{1}, l_{2}}^{ \pm}= \pm \partial_{\beta}+\left(l_{2}+1 / 2\right) \tanh \beta+\left(-l_{1}+1 / 2\right) \operatorname{coth} \beta, \quad \lambda_{l_{1}, l_{2}}=-\left(1-l_{1}+l_{2}\right)^{2} .
$$

The operators $C_{l_{1}, l_{2}}^{ \pm}$give rise to the intertwining relations

$$
C_{l_{1}+1, l_{2}-1}^{+} H_{l_{1}, l_{2}}^{\beta}=H_{l_{1}+1, l_{2}-1}^{\beta} C_{l_{1}+1, l_{2}-1}^{+}, \quad C_{l_{1}+1, l_{2}-1}^{-} H_{l_{1}+1, l_{2}-1}^{\beta}=H_{l_{1}, l_{2}}^{\beta} C_{l_{1}+1, l_{2}-1}^{-},
$$

which imply the connection among eigenfunctions

$$
C_{l_{1}+1, l_{2}-1}^{-}: f_{l_{1}+1, l_{2}-1} \rightarrow f_{l_{1}, l_{2}}, \quad C_{l_{1}+1, l_{2}-1}^{+}: f_{l_{1}, l_{2}} \rightarrow f_{l_{1}+1, l_{2}-1} .
$$

The IO's $C_{l_{1}, l_{2}}^{ \pm}$can also be expressed in terms of the first set of coordinates $(\xi, \theta)$

$$
C_{l_{1}, l_{2}}^{ \pm}= \pm\left(\sin \theta \partial_{\xi}+\cos \theta \operatorname{coth} \xi \partial_{\theta}\right)+\left(l_{2}+1 / 2\right) \tanh \xi \sin \theta+\left(-l_{1}+1 / 2\right) \operatorname{coth} \xi \csc \theta .
$$

New free-index operators are defined as

$$
\hat{C}^{-} f_{l_{1}, l_{2}}:=\frac{1}{2} C_{l_{1}, l_{2}}^{-} f_{l_{1}, l_{2}}, \quad \hat{C}^{+} f_{l_{1}, l_{2}}:=\frac{1}{2} C_{l_{1}, l_{2}}^{+} f_{l_{1}, l_{2}}, \quad \hat{C} f_{l_{1}, l_{2}}:=-\frac{1}{2}\left(l_{2}-l_{1}\right) f_{l_{1}, l_{2}},
$$

satisfying the commutation relations of the $s u(1,1)$ algebra

$$
\left[\hat{C}^{-}, \hat{C}^{+}\right]=2 \hat{C}, \quad\left[\hat{C}, \hat{C}^{ \pm}\right]= \pm \hat{C}^{ \pm} .
$$


The fundamental state for the $s u(1,1)$ representation, given by $\hat{C}^{-} f_{l_{1}, l_{2}}^{0}=0$, has the expression

$$
f_{l_{1}, l_{2}}^{0}(\beta)=N(\cosh \beta)^{l_{2}+1 / 2}(\sinh \beta)^{-l_{1}+1 / 2},
$$

with $N$ a normalization constant. In order to get an IUR from this eigenfunction, we impose it to be regular and normalizable, therefore

$$
l_{1} \leq 1 / 2, \quad-k_{2} \equiv l_{2}-l_{1}<-1 .
$$

The lowest weight of the IUR is given by

$$
j_{2}^{\prime}=k_{2} / 2>1 / 2
$$

because in this case we have that $\hat{C} f_{l_{1}, l_{2}}^{0}=-\frac{1}{2}\left(l_{2}-l_{1}\right) f_{l_{1}, l_{2}}^{0}$.

As in the other cases the IO's $C^{ \pm}$can be considered as connecting global Hamiltonians $H_{\ell}$ and their eigenfunctions, having in mind that now the parameter $l_{0}$ is unaltered.

\subsubsection{Algebraic structure of the intertwining operators}

If we consider together all the IO's $\left\{\hat{A}^{ \pm}, \hat{A}, \hat{B}^{ \pm}, \hat{B}, \hat{C}^{ \pm}, \hat{C}\right\}$ we find that they close a $s u(2,1)$ Lie algebra, whose Lie commutators are displayed in (9), (13) and (14) together with the crossed commutators

$$
\begin{array}{lll}
{\left[\hat{A}^{+}, \hat{B}^{+}\right]=0,} & {\left[\hat{A}^{+}, \hat{B}^{-}\right]=-\hat{C}^{-},} & {\left[\hat{A}^{+}, \hat{B}\right]=-\frac{1}{2} \hat{A}^{+},} \\
{\left[\hat{A}^{+}, \hat{C}^{+}\right]=\hat{B}^{+},} & {\left[\hat{A}^{+}, \hat{C}^{-}\right]=0,} & {\left[\hat{A}^{+}, \hat{C}\right]=\frac{1}{2} \hat{A}^{+},} \\
{\left[\hat{A}^{-}, \hat{B}^{+}\right]=\hat{C}^{+},} & {\left[\hat{A}^{-}, \hat{B}^{-}\right]=0,} & {\left[\hat{A}^{-}, \hat{B}\right]=\frac{1}{2} \hat{A}^{-},} \\
{\left[\hat{A}^{-}, \hat{C}^{+}\right]=0,} & {\left[\hat{A}^{-}, \hat{C}^{-}\right]=-\hat{B}^{-},} & {\left[\hat{A}^{-}, \hat{C}\right]=-\frac{1}{2} \hat{A}^{-},} \\
{\left[\hat{A}, \hat{B}^{+}\right]=\frac{1}{2} \hat{B}^{+},} & {\left[\hat{A}, \hat{B}^{-}\right]=-\frac{1}{2} \hat{B}^{-},} & {[\hat{A}, \hat{B}]=0,} \\
{\left[\hat{A}, \hat{C}^{+}\right]=-\frac{1}{2} \hat{C}^{+},} & {\left[\hat{A}, \hat{C}^{-}\right]=\frac{1}{2} \hat{C}^{-},} & {[\hat{A}, \hat{C}]=0,} \\
{\left[\hat{B}^{+}, \hat{C}^{+}\right]=0,} & {\left[\hat{B}^{+}, \hat{C}^{-}\right]=-\hat{A}^{+},} & {[\hat{B}+\hat{C}]=-\frac{1}{2} \hat{B}^{+},} \\
{\left[\hat{B}^{-}, \hat{C}^{+}\right]=\hat{A}^{-},} & {\left[\hat{B}^{-}, \hat{C}^{-}\right]=0,} & {[\hat{B}-\hat{C}]=\frac{1}{2} \hat{B}^{-},} \\
{\left[\hat{B}, \hat{C}^{+}\right]=\frac{1}{2} \hat{C}^{+},} & {\left[\hat{B}, \hat{C}^{-}\right]=-\frac{1}{2} \hat{C}^{-},} & {[\hat{B}, \hat{C}]=0 .}
\end{array}
$$

The second order Casimir operator of $s u(2,1)$ is

$$
\mathcal{C}=\hat{A}^{+} \hat{A}^{-}-\hat{B}^{+} \hat{B}^{-}-\hat{C}^{+} \hat{C}^{-}+\frac{2}{3}\left(\hat{A}^{2}+\hat{B}^{2}+\hat{C}^{2}\right)-(\hat{A}+\hat{B}+\hat{C}) .
$$

Note that in our differential realization

$$
\hat{A}-\hat{B}+\hat{C}=0
$$

and that there is another generator,

$$
\mathcal{C}^{\prime}=l_{1}+l_{2}-l_{0},
$$


commuting with the rest of generators of $s u(2,1)$. Hence

$$
\left\langle\hat{A}^{ \pm}, \hat{A}, \hat{B}^{ \pm}, \hat{B}, \hat{C}^{ \pm}, \hat{C}\right\rangle \oplus\left\langle\mathcal{C}^{\prime}\right\rangle \approx u(2,1) .
$$

The Hamiltonian (6) can be rewritten in terms of $\mathcal{C}$ and $\mathcal{C}^{\prime}$ as

$$
\begin{aligned}
H_{\ell} & =-4 \mathcal{C}+\frac{1}{3} \mathcal{C}^{\prime 2}-\frac{15}{4} \\
& =-4\left(\hat{A}^{+} \hat{A}^{-}-\hat{B}^{+} \hat{B}^{-}-\hat{C}^{+} \hat{C}^{-}+\frac{2}{3}\left(\hat{A}^{2}+\hat{B}^{2}+\hat{C}^{2}\right)-(\hat{A}+\hat{B}+\hat{C})\right)+\frac{1}{3} \mathcal{C}^{\prime 2}-\frac{15}{4} .
\end{aligned}
$$

The quadratic operators $\hat{A}^{+} \hat{A}^{-}, \hat{B}^{+} \hat{B}^{-}$and $\hat{C}^{+} \hat{C}^{-}$commute with the Hamiltonian and they are constants of motion. However, they do not commute among themselves giving cubic expressions

$$
\left[\hat{A}^{+} \hat{A}^{-}, \hat{B}^{+} \hat{B}^{-}\right]=-\left[\hat{A}^{+} \hat{A}^{-}, \hat{C}^{+} \hat{C}^{-}\right]=-\left[\hat{B}^{+} \hat{B}^{-}, \hat{C}^{+} \hat{C}^{-}\right]=\hat{A}^{+} \hat{C}^{+} \hat{B}^{-}-\hat{B}^{+} \hat{C}^{-} \hat{A}^{-} .
$$

However, as we will see later, they generate a quadratic algebra.

The eigenfunctions of the Hamiltonians $H_{\ell}$, that have the same energy, support an IUR of $s u(2,1)$ characterized by a value of $\mathcal{C}$ and other of $\mathcal{C}^{\prime}$. These representations can be obtained, as usual, starting from a fundamental state simultaneously annihilated by the lowering operators $\hat{A}^{-}, \hat{C}^{-}$and $\hat{B}^{-}$

$$
\hat{A}_{\ell}^{-} \Phi_{\ell}^{0}=\hat{C}_{\ell}^{-} \Phi_{\ell}^{0}=\hat{B}_{\ell}^{-} \Phi_{\ell}^{0}=0 .
$$

Solving these equations we find

$$
\Phi_{\ell}^{0}(\xi, \theta)=N(\cos \theta)^{l_{0}+1 / 2}(\sin \theta)^{1 / 2}(\cosh \xi)^{l_{2}+1 / 2}(\sinh \xi)^{l_{0}+1},
$$

where $\ell=\left(l_{0}, 0, l_{2}\right)$ and $N$ is a normalization constant. From previous inequalities the parameters $l_{0}, l_{2}$ of $\Phi_{\ell}^{0}$ must satisfy

$$
\left(l_{0}+l_{2}\right)<-3 / 2, \quad l_{0} \geq-1 / 2 .
$$

In order to guarantee the normalization of $\Phi_{\ell}^{0}$ using the invariant measure the values of the parameters $l_{0}$ and $l_{2}$ have to verify

$$
\left(l_{0}+l_{2}\right)<-5 / 2 .
$$

Note that the state $\Phi_{\ell}^{0}$ supports also IUR's of the subalgebras $s u(2)$ (generated by $\hat{A}^{ \pm}$with the weight $\left.j=l_{0} / 2\right)$ and $s u(1,1)$ (generated by $\hat{C}^{ \pm}$with $j_{2}^{\prime}=-l_{2} / 2$ ).

The energies of the fundamental states $\Phi_{\ell}^{0}(\xi, \theta)$ are obtained from $H_{\ell}$ taking into account the expressions for the Casimir operators $\mathcal{C}$ and $\mathcal{C}^{\prime}$

$$
H_{\ell} \Phi_{\ell}^{0}=-\left(l_{0}+l_{2}+3 / 2\right)\left(l_{0}+l_{2}+5 / 2\right) \Phi_{\ell}^{0} \equiv E_{\ell}^{0} \Phi_{\ell}^{0} .
$$

From $\Phi_{\ell}^{0}$ we can get the other eigenfunctions in the $s u(2,1)$ representation using the raising operators $\hat{A}^{+}, \hat{B}^{+}, \hat{C}^{+}$, all of them sharing the same energy eigenvalue $E_{\ell}^{0}$. Notice that the expression for $E_{\ell}^{0}$ depends on $l_{0}+l_{2}$, hence the states in the family of IUR's derived from fundamental states $\Phi_{\ell}^{0}(\xi, \theta)$, sharing the same value of $l_{0}+l_{2}$, also shall have the same energy eigenvalue. The energy $E_{\ell}^{0}$ corresponding to bound states is negative and the set of such bound states for each Hamiltonian $H_{\ell}$ is finite.

In Fig. 1 we display the states of some IUR's of $s u(2,1)$ by points $\left(l_{0}, l_{1}, l_{2}\right) \in \mathbb{R}^{3}$ linked to the ground state $\Phi_{\ell}^{0}$, characterized by $\left(l_{0}, 0, l_{2}\right)$, by the raising operators $\hat{A}^{+}$and $\hat{C}^{+}$. The points associated to a IUR are in a $2 \mathrm{D}$ plane (fixed by the particular value $l_{0}+l_{2}=-3$ of $\mathcal{C}^{\prime}$ ) and, 


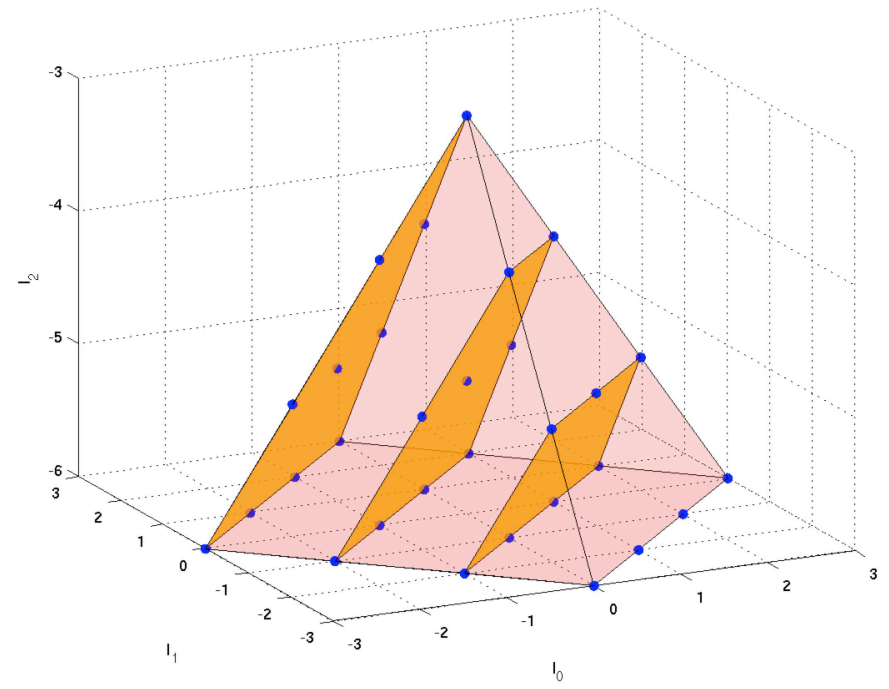

Figure 1. States of IUR's of $s u(2,1)$ sharing the same energy and represented by points in the three dark planes associated to $\Phi_{\ell}^{0}$ with $\ell=(0,0,-3), \ell=(1,0,-4)$ and $\ell=(2,0,-5)$.

obviously, the other IUR's are described by points in parallel 2D planes. These parallel planes are placed inside a tetrahedral unbounded pyramid whose basis extends towards $-\infty$ along the axis $l_{2}$.

On the other hand, there exist some points (in the parameter space of parameters $\left(l_{0}, l_{1}, l_{2}\right)$ ) which are degenerated because they correspond to an eigenspace with dimension bigger than 1 . For instance, let us consider the representation characterized by the fundamental state $\Phi_{\ell}^{0}$ where $\ell=(0,0,-3)$ : its points lie in a triangle and are nondegenerated. The IUR corresponding to the ground state with $\ell^{\prime}=(1,0,-4)$ has eigenstates with the same energy, $E=-(-3+3 / 2)(-3+5 / 2)$, as the previous one, since both share the same value of $l_{0}+l_{2}=-3$. The eigenstates corresponding to $\ell^{\prime \prime}=(0,0,-5)$, inside this representation, may be obtained in two ways:

$$
\Phi_{(0,0,-5)}^{2}=\hat{C}^{+} \hat{A}^{+} \Phi_{(0,0,-3)}^{0}, \quad \tilde{\Phi}_{(0,0,-5)}^{2}=\hat{A}^{+} \hat{C}^{+} \Phi_{(0,0,-3)}^{0} .
$$

We have two independent states spanning a 2-dimensional eigenspace of the Hamiltonian $H_{(0,0,-5)}$ for that eigenvalue of the energy $E=-(-3+3 / 2)(-3+5 / 2)$. The ground state for $H_{(0,0,-5)}$ is given by the wavefunction $\Phi_{(0,0,-5)}^{0}$ and its energy is $E_{(0,0,-5)}^{0}=-(-5+3 / 2)(-5+5 / 2)$.

In a similar way it is possible to obtain the degeneration of higher excited levels in the discrete spectrum of the Hamiltonians. Thus, the $n$-excited level, when it exists, has associated an $n$-dimensional eigenspace.

\subsubsection{The complete symmetry algebra $s o(4,2)$}

By simple inspection one can see that the Hamiltonian $H_{\ell}(6)$ is invariant under reflections in the space of parameters $\left(l_{0}, l_{1}, l_{2}\right)$

$$
\begin{aligned}
& I_{0}:\left(l_{0}, l_{1}, l_{2}\right) \rightarrow\left(-l_{0}, l_{1}, l_{2}\right), \quad I_{1}:\left(l_{0}, l_{1}, l_{2}\right) \rightarrow\left(l_{0},-l_{1}, l_{2}\right), \\
& I_{2}:\left(l_{0}, l_{1}, l_{2}\right) \rightarrow\left(l_{0}, l_{1},-l_{2}\right) .
\end{aligned}
$$

These operators generate by conjugation other sets of intertwining operators from the ones already defined. Thus,

$$
I_{0}:\left\{\hat{A}^{ \pm}, \hat{A}\right\} \longrightarrow\left\{\tilde{A}^{ \pm}=I_{0} \hat{A}^{ \pm} I_{0}, \tilde{A}=I_{0} \hat{A} I_{0}\right\},
$$


where

$$
\tilde{A}_{l_{0}, l_{1}}^{ \pm}= \pm \partial_{\theta}-\left(-l_{0}+1 / 2\right) \tan \theta+\left(l_{1}+1 / 2\right) \cot \theta, \quad \tilde{\lambda}_{l_{0}, l_{1}}=\left(1-l_{0}+l_{1}\right)^{2} .
$$

They act on the eigenfunctions of the Hamiltonians (7) in the following way

$$
\tilde{A}_{l_{0}, l_{1}}^{-}: f_{l_{0}, l_{1}} \rightarrow f_{l_{0}-1, l_{1}+1}, \quad \tilde{A}_{l_{0}, l_{1}}^{+}: f_{l_{0-1}, l_{1}+1} \rightarrow f_{l_{0}, l_{1}} .
$$

In these conditions, we can define global operators $\tilde{A}^{ \pm}$as we made before. Then, $\tilde{A}^{ \pm}$together with $\tilde{A} f_{l_{0}, l_{1}}:=-\frac{1}{2}\left(-l_{0}+l_{1}\right) f_{l_{0}, l_{1}}$ close a second $\widetilde{s u}(2)$.

In a similar way new sets of operators $\left\{\tilde{B}^{ \pm}, \tilde{B}\right\}$ and $\left\{\tilde{C}^{ \pm}, \tilde{C}\right\}$ closing $\widetilde{s u}(1,1)$ algebras, can also be defined

$$
\begin{aligned}
& I_{0}:\left\{A^{ \pm}, A ; B^{ \pm}, B ; C^{ \pm}, C\right\} \longrightarrow\left\{\tilde{A}^{ \pm}, \tilde{A} ; \tilde{B}^{ \pm}, \tilde{B} ; C^{ \pm}, C\right\} \\
& I_{1}:\left\{A^{ \pm}, A ; B^{ \pm}, B ; C^{ \pm}, C\right\} \longrightarrow\left\{\tilde{A}^{\mp},-\tilde{A} ; B^{ \pm}, B ; \tilde{C}^{ \pm}, \tilde{C}\right\} \\
& I_{2}:\left\{A^{ \pm}, A ; B^{ \pm}, B ; C^{ \pm}, C\right\} \longrightarrow\left\{A^{ \pm}, A ; \tilde{B}^{\mp},-\tilde{B} ;-\tilde{C}^{\mp},-\tilde{C}\right\} .
\end{aligned}
$$

The whole set of the operators $\left\{A^{ \pm}, \tilde{A}^{ \pm}, B^{ \pm}, \tilde{B}^{ \pm}, C^{ \pm}, \tilde{C}^{ \pm}\right\}$together with the set of diagonal operators $\left\{L_{0}, L_{1}, L_{2}\right\}$, defined by

$$
L_{i} \Psi_{\ell}=l_{i} \Psi_{\ell}
$$

span a Lie algebra of rank three: $o(4,2)$. The Lie commutators of $o(4,2)$ can be easily derived from those of $s u(2,1)$ and the action of the reflections. It is obvious, by construction, that all these generators link eigenstates of Hamiltonians $H_{\ell}$ with the same eigenvalue.

The fundamental state $\Psi_{\ell}^{0}$ for $s o(4,2)$ will be annihilated by all the lowering operators

$$
A_{\ell}^{-} \Psi_{\ell}^{0}=\tilde{A}_{\ell}^{-} \Psi_{\ell}^{0}=C_{\ell}^{-} \Psi_{\ell}^{0}=\tilde{C}_{\ell}^{-} \Psi_{\ell}^{0}=B_{\ell}^{-} \Psi_{\ell}^{0}=\tilde{B}_{\ell}^{-} \Psi_{\ell}^{0}=0 .
$$

This state should be a particular case of the state given by expression (15), i.e. it should be also invariant under the $l_{0}$-reflection,

$$
\Phi_{\left(l_{0}=0, l_{1}=0, l_{2}\right)}^{0}(\xi, \theta)=N(\cos \theta)^{1 / 2}(\sin \theta)^{1 / 2}(\cosh \xi)^{l_{2}+1 / 2} \sinh \xi
$$

where $l_{2}<-5 / 2$. This point $\left(l_{0}=0, l_{1}=0, l_{2}\right)$ in the parameter space, for the cases displayed in Fig. 1, corresponds to the top vertex of the pyramid, from which all the other points plotted can be obtained with the help of raising operators. Such points correspond to an IUR of $s o(4,2)$ algebra that includes the series of IUR's of $s u(2,1)$.

Fixed the IUR of $s o(4,2)$ corresponding to a value of $\ell=\left(0,0, l_{2}\right)$ such that $-7 / 2 \leq l_{2}<-5 / 2$, then the points on the surface of the associated pyramid in the parameter space correspond to non-degenerated ground levels of their respective Hamiltonians. This 'top' pyramid includes inside other 'lower' pyramids with vertexes at the points $\ell_{n}=\left(0,0, l_{2}-2 n\right)$. Each point on the surface of an inner pyramid associated to $\ell_{n}$ represents an $n$-excited level $n$-fold degenerated of the IUR associated to $\ell$ (see Fig. 2).

\subsection{Superintegrable quantum $u(3)$-system}

In this case we consider the quantum Hamiltonian

$$
H_{\ell}=-\left(J_{0}^{2}+J_{1}^{2}+J_{2}^{2}\right)+\frac{l_{0}^{2}-1 / 4}{\left(s_{0}\right)^{2}}+\frac{l_{1}^{2}-1 / 4}{\left(s_{1}\right)^{2}}+\frac{l_{2}^{2}-1 / 4}{\left(s_{2}\right)^{2}},
$$




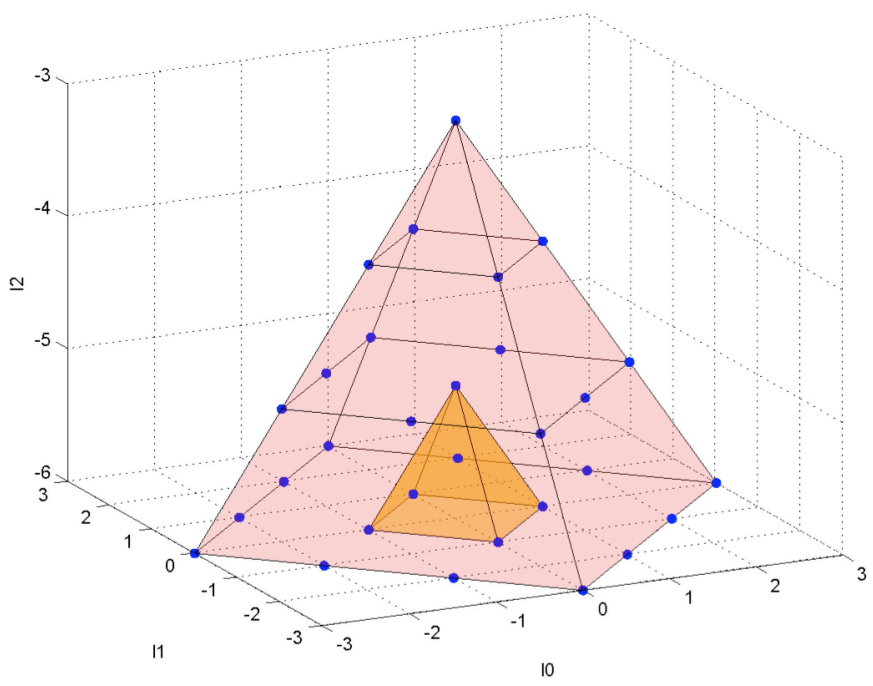

Figure 2. Two pyramids associated to the same IUR of $s o(4,2)$. The points of the faces of the exterior pyramid (with vertex $(0,0,-3))$ represent non-degenerated levels. The exterior faces of the inner pyramid (vertex $(0,0,-5))$ are first excited double-degenerated levels.

where $\ell=\left(l_{0}, l_{1}, l_{2}\right) \in \mathbb{R}^{3}, J_{i}=-\epsilon_{i j k} s_{j} \partial_{k}(i=0,1,2)$ and its configuration space is the 2 -sphere

$$
\mathcal{S}^{2} \equiv\left(s_{0}\right)^{2}+\left(s_{1}\right)^{2}+\left(s_{2}\right)^{2}=1, \quad\left(s_{0}, s_{1}, s_{2}\right) \in \mathbb{R}^{3} .
$$

In spherical coordinates

$$
s_{0}=\cos \phi \cos \theta, \quad s_{1}=\cos \phi \sin \theta, \quad s_{2}=\sin \phi, \quad \phi \in[-\pi / 2, \pi / 2], \quad \theta \in[0,2 \pi],
$$

that parametrize $\mathcal{S}^{2}$, the eigenvalue problem $H_{\ell} \Psi=E \Psi$ takes the expression

$$
\left[-\partial_{\phi}^{2}+\tan \phi \partial_{\phi}+\frac{l_{2}^{2}-1 / 4}{\sin ^{2}(\phi)}+\frac{1}{\cos ^{2} \phi}\left[-\partial_{\theta}^{2}+\frac{l_{0}^{2}-1 / 4}{\cos ^{2} \theta}+\frac{l_{1}^{2}-1 / 4}{\sin ^{2} \theta}\right]\right] \Psi=E \Psi .
$$

Taking solutions separated in the variables $\theta$ and $\phi$ as $\boldsymbol{\Psi}(\theta, \phi)=f(\theta) g(\phi)$ we find

$$
\begin{aligned}
& H_{l_{0}, l_{1}}^{\theta} f(\theta) \equiv\left[-\partial_{\theta}^{2}+\frac{l_{0}^{2}-1 / 4}{\cos ^{2} \theta}+\frac{l_{1}^{2}-1 / 4}{\sin ^{2} \theta}\right] f(\theta)=\alpha f(\theta), \\
& {\left[-\partial_{\phi}^{2}+\tan \phi \partial_{\phi}+\frac{\alpha}{\cos ^{2} \phi}+\frac{l_{2}^{2}-1 / 4}{\sin ^{2} \phi}\right] g(\phi)=E g(\phi),}
\end{aligned}
$$

with $\alpha>0$ a separating constant. Note that equation (19) is equal to equation (7) corresponding to the $s u(2,1)$ case.

Following the procedure of the previous case of $s o(2,1)$ we can factorize the Hamiltonian (19) in terms of operators $A_{n}^{ \pm}$like those of expression (8), obtaining, finally, a su(2) algebra.

We can find other two sets of spherical coordinates, that parametrize the sphere $\mathcal{S}^{2}$ and that separate the Hamiltonian (19), Thus, we get two new sets of intertwining operators $B_{n}^{ \pm}$ and $C_{n}^{ \pm}$, like in the $s u(2,1)$ case. In this way, we can construct an algebra $u(3)$ and using reflection operators, acting in the space of the parameters of the Hamiltonian (17), this algebra is enlarged to $s o(6)$.

These three sets of operators are related, as we saw in Section 3.1, with three sets of (spherical) coordinates that we can take in the 2 -sphere immersed in a 3-dimensional ambient space 
with cartesian axes $\left\{s_{0}, s_{1}, s_{2}\right\}$. Since the coordinates $\left(s_{0}, s_{1}, s_{2}\right)$ play a symmetric role in the Hamiltonian (17), we will take their cyclic rotations to get two other intertwining sets. Thus, we take the spherical coordinates choosing as 'third axis' $s_{1}$ instead of $s_{2}$ as in (18),

$$
s_{2}=\cos \psi \cos \xi, \quad s_{0}=\cos \psi \sin \xi, \quad s_{1}=\sin \psi, \quad \psi \in[-\pi / 2, \pi / 2], \quad \xi \in[0,2 \pi] .
$$

The corresponding intertwining operators $B_{\left(l_{0}, l_{1}, l_{2}\right)}^{ \pm}$are defined in a similar way to $A_{\left(l_{0}, l_{1}, l_{2}\right)}^{ \pm}$. The explicit expressions for the new set in terms of the initial coordinates $(\theta, \phi)(18)$ are

$$
B_{\left(l_{0}, l_{1}, l_{2}\right)}^{ \pm}= \pm\left(\sin \theta \tan \phi \partial_{\theta}+\cos \theta \partial_{\phi}\right)-\left(l_{2}+1 / 2\right) \cos \theta \cot \phi+\left(l_{0}+1 / 2\right) \sec \theta \tan \phi .
$$

The spherical coordinates around the $s_{0}$ axis are

$$
s_{1}=\cos \beta \cos \eta, \quad s_{2}=\cos \beta \sin \eta, \quad s_{0}=\sin \beta .
$$

We obtain a new pair of operators, that written in terms of the original variables $(\theta, \phi)$ are

$$
C_{\left(l_{0}, l_{1}, l_{2}\right)}^{ \pm}= \pm\left(\cos \theta \tan \phi \partial_{\theta}-\sin \theta \partial_{\phi}\right)+\left(l_{1}-1 / 2\right) \operatorname{cosec} \theta \tan \phi+\left(l_{2}+1 / 2\right) \sin \theta \cot \phi .
$$

They intertwine the Hamiltonians in the following way

$$
C_{\left(l_{0}, l_{1}, l_{2}\right)}^{-} H_{\left(l_{0}, l_{1}, l_{2}\right)}=H_{\left(l_{0}, l_{1}-1, l_{2}+1\right)} C_{\left(l_{0}, l_{1}, l_{2}\right)}^{-}, \quad C_{\left(l_{0}, l_{1}, l_{2}\right)}^{+} H_{\left(l_{0}, l_{1}-1, l_{2}+1\right)}=H_{\left(l_{0}, l_{1}, l_{2}\right)} C_{\left(l_{0}, l_{1}, l_{2}\right)}^{+} .
$$

The free-index or 'global' operators close a third $s u(2)$.

All these transformations $\left\{A^{ \pm}, A, B^{ \pm}, B, C^{ \pm}, C\right\}$ (where $A-B+C=0$ ) span an algebra $s u(3)$, whose Lie commutators are

$$
\begin{array}{lll}
{\left[\hat{A}^{+}, \hat{A}^{-}\right]=2 A,} & {\left[\hat{A}, \hat{A}^{ \pm}\right]= \pm \hat{A}^{ \pm},} & {\left[\hat{B}^{+}, \hat{B}^{-}\right]=2 B,} \\
{\left[\hat{B}, \hat{B}^{ \pm}\right]= \pm \hat{B}^{ \pm},} & {\left[\hat{C}, \hat{C}^{ \pm}\right]= \pm \hat{C}^{ \pm},} & {\left[\hat{C}^{+}, \hat{C}^{-}\right]=2 C,} \\
{\left[\hat{A}^{+}, \hat{B}^{+}\right]=0,} & {\left[\hat{A}^{+}, \hat{B}^{-}\right]=\hat{C}^{-},} & {\left[\hat{A}^{+}, \hat{B}\right]=-\frac{1}{2} \hat{A}^{+},} \\
{\left[\hat{A}^{+}, \hat{C}^{+}\right]=-\hat{B}^{+},} & {\left[\hat{A}^{+}, \hat{C}^{-}\right]=0,} & {\left[\hat{A}^{+}, \hat{C}\right]=\frac{1}{2} \hat{A}^{+},} \\
{\left[\hat{A^{-},} \hat{B}^{+}\right]=-\hat{C}^{+},} & {\left[\hat{A}^{-}, \hat{B}^{-}\right]=0,} & {\left[\hat{A}^{-}, \hat{B}\right]=\frac{1}{2} \hat{A}^{-},} \\
{\left[\hat{A}^{-}, \hat{C}^{+}\right]=0,} & {\left[\hat{A}^{-}, \hat{C}^{-}\right]=\hat{B}^{-},} & {\left[\hat{A}^{-}, \hat{C}\right]=-\frac{1}{2} \hat{A}^{-},} \\
{\left[\hat{A}, \hat{B}^{+}\right]=\frac{1}{2} \hat{B}^{+},} & {\left[\hat{A}, \hat{B}^{-}\right]=-\frac{1}{2} \hat{B}^{-},} & {[\hat{A}, \hat{B}]=0,} \\
{\left[\hat{A}, \hat{C}^{+}\right]=-\frac{1}{2} \hat{C}^{+},} & {\left[\hat{A}, \hat{C}^{-}\right]=\frac{1}{2} \hat{C}^{-},} & {[\hat{A}, \hat{C}]=0,} \\
{\left[\hat{B}+, \hat{C}^{+}\right]=0,} & {\left[\hat{B}^{+}, \hat{C}^{-}\right]=-\hat{A}^{+},} & {[\hat{B}+\hat{C}]=-\frac{1}{2} \hat{B}^{+},} \\
{\left[\hat{B}-, \hat{C}^{+}\right]=\hat{A}^{-},} & {\left[\hat{B}^{-}, \hat{C}^{-}\right]=0,} & {[\hat{B}-\hat{C}]=\frac{1}{2} \hat{B}^{-},} \\
{\left[\hat{B}, \hat{C}^{+}\right]=\frac{1}{2} \hat{C}^{+},} & {\left[\hat{B}, \hat{C}^{-}\right]=-\frac{1}{2} \hat{C}^{-},} & {[\hat{B}, \hat{C}]=0 .}
\end{array}
$$

The second order Casimir operator of $s u(3)$ is given by

$$
\mathcal{C}=A^{+} A^{-}+B^{+} B^{-}+C^{+} C^{-}+\frac{2}{3} A(A-3 / 2)+\frac{2}{3} B(B-3 / 2)+\frac{2}{3} C(C-3 / 2) .
$$

We obtain a $u(3)$ algebra by adding the central diagonal operator

$$
D:=l_{0}-l_{1}-l_{2} \text {. }
$$


The global operator convention can be adopted for the Hamiltonians in the $u(3)$-hierarchy by defining its action on the eigenfunctions $\Phi_{\left(l_{1}, l_{2}, l_{3}\right)}$ of $H_{\left(l_{1}, l_{2}, l_{3}\right)}$ by $H \Phi_{\left(l_{1}, l_{2}, l_{3}\right)}:=H_{\left(l_{1}, l_{2}, l_{3}\right)} \Phi_{\left(l_{1}, l_{2}, l_{3}\right)}$. Then, $H$ can be expressed in terms of both Casimir operators, (20) and (21), as

$$
H=4 \mathcal{C}-\frac{1}{3} D^{2}+\frac{15}{4} .
$$

Hence, the Hamiltonian can be written as a certain quadratic function of the operators $A^{ \pm}, B^{ \pm}$ and $C^{ \pm}$generalizing the usual factorization for one-dimensional systems plus a constant since in the representation that we are using the operators $A, B, C$ are diagonal depending on the parameters $l_{0}, l_{1}, l_{2}$,

$$
H=4\left(A^{+} A^{-}+B^{+} B^{-}+C^{+} C^{-}\right)+\text {cnt. }
$$

The quadratic operators $A^{+} A^{-}, B^{+} B^{-}, C^{+} C^{-}$commute with $H$ but do not commute among themselves

$$
\left[A^{+} A^{-}, B^{+} B^{-}\right]=-\left[A^{+} A^{-}, C^{+} C^{-}\right]=\left[B^{+} B^{-}, C^{+} C^{-}\right]=-A^{+} C^{+} B^{-}+B^{+} C^{-} A^{-} .
$$

The intertwining operators can help also in supplying the elementary integrals of motion. We have two kinds of integrals: (i) second order constants, defined by the quadratic operators $X_{1}=A^{+} A, X_{2}=B^{+} B, X_{3}=C^{+} C$; and (ii) third order constants defined by cubic operators: $Y_{1}=A^{+} C^{+} B^{-}, Y_{2}=B^{+} C^{-} A^{-}=\left(Y_{1}\right)^{+}$. Of course, since this system is superintegrable, there are only three functionally independent constants of motion, for instance $X_{1}, X_{2}, X_{3}$. This set of symmetries $\left\{X_{i}, Y_{j}\right\}$ closes a quadratic algebra, as it is well known from many references $[3,18,25,26,27,28]$. The commutators in this case are

$$
\begin{aligned}
& {\left[X_{1}, X_{2}\right]=-\left[X_{1}, X_{3}\right]=\left[X_{2}, X_{3}\right]=-Y_{1}+Y_{2},} \\
& {\left[X_{1}, Y_{1}\right]=X_{1} X_{2}-X_{1} X_{3}-2(A-1) Y_{1}} \\
& {\left[X_{1}, Y_{2}\right]=-X_{2} X_{1}+X_{3} X_{1}+2(A-1) Y_{2},} \\
& {\left[X_{2}, Y_{1}\right]=X_{1} X_{2}-X_{2} X_{3}-(1+2 B) Y_{1}+Y_{2}-2 C X_{2},} \\
& {\left[X_{2}, Y_{2}\right]=-X_{2} X_{1}+X_{3} X_{2}+(1+2 B) Y_{2}-Y_{1}+2 C X_{2},} \\
& {\left[X_{3}, Y_{1}\right]=-X_{1} X_{3}-X_{2} X_{3}+2 C Y_{1}-2 C X_{2}+Y_{2},} \\
& {\left[X_{3}, Y_{2}\right]=X_{3} X_{1}+X_{3} X_{2}-2 C Y_{2}+2 C X_{2}-Y_{1},} \\
& {\left[Y_{1}, Y_{2}\right]=2\left(-C X_{1} X_{2}+B X_{1} X_{3}+A X_{2} X_{3}+(B+C) Y_{1}-A Y_{2}+2 A C X_{2}\right) .}
\end{aligned}
$$

Remark that the operators $\{A, B, C\}$ are diagonal with fixed values for each Hamiltonian.

One can show that the eigenstates of this Hamiltonian hierarchy are connected to the IUR's of $u(3)$. Fundamental states $\Phi$ annihilated by $A^{-}$and $C^{-}$(simple roots of $\left.s u(3)\right)$,

$$
A_{\ell}^{-} \Phi_{\ell}=C_{\ell}^{-} \Phi_{\ell}=0
$$

only exist when $l_{1}=0$. Their explicit form is

$$
\Phi_{\ell}(\theta, \phi)=N \cos ^{l_{0}+1 / 2} \theta \sin ^{1 / 2} \theta \cos ^{l_{0}+1} \phi \sin ^{l_{2}+1 / 2} \phi,
$$

whit $N$ a normalizing constant. The diagonal operators act on them as

$$
\begin{array}{ll}
A \Phi_{\ell}=-l_{0} / 2 \Phi_{\ell}, & l_{0}=m, \quad l_{1}=0, \quad m=0,1,2, \ldots \\
C \Phi_{\ell}=-l_{2} / 2 \Phi_{\ell}, & l_{2}=n, \quad n=0,1,2, \ldots
\end{array}
$$

This shows that $\Phi_{\ell}$ is the lowest state of the IUR $j_{1}=m / 2$ of the subalgebra $s u(2)$ generated by $\left\{A^{ \pm}, A\right\}$, and of the IUR $j_{2}=n / 2$ of the subalgebra $s u(2)$ spanned by $\left\{C^{ \pm}, C\right\}$. Such 
a $s u(3)$-representation will be denoted $(m, n), m, n \in \mathbb{Z} \geq 0$. The points (labelling the states) of this representation obtained from $\Phi_{\ell}$ lie on the plane $D=m-n$ inside the $\ell$-parameter space.

The energy for the states of the IUR, determined by the lowest state (23) with parameters $\left(l_{0}, 0, l_{2}\right)$, is given $(22)$ by

$$
E=\left(l_{0}+l_{2}+3 / 2\right)\left(l_{0}+l_{2}+5 / 2\right)=(m+n+3 / 2)(m+n+5 / 2) .
$$

Note that the IUR's labelled by $(m, n)$ with the same value $m+n$ are associated to states with the same energy (iso-energy representations). This degeneration will be broken using so(6).

Making use of some relevant discrete symmetries, following the procedure of Section 3.1.6, the dynamical algebra $u(3)$ can be enlarged to so(6).

The Hamiltonian $H_{\left(l_{0}, l_{1}, l_{2}\right)}(17)$ is invariant under reflections (16) in the parameter space $\left(l_{0}, l_{1}, l_{2}\right)$. These symmetries, $I_{i}$, can be directly implemented in the eigenfunction space, giving by conjugation another set of intertwining operators $\left\{\tilde{X}=I_{i} X I_{i}, i=0,1,2\right\}$ closing an isomorphic Lie algebra $\tilde{u}(3)$. They are

$$
\begin{aligned}
& \left\{A^{ \pm}, B^{ \pm}, C^{ \pm}\right\} \stackrel{I_{0}}{\longrightarrow}\left\{\tilde{A}^{\mp}, \tilde{B}^{\mp}, C^{ \pm}\right\}, \\
& \left\{A^{ \pm}, B^{ \pm}, C^{ \pm}\right\} \stackrel{I_{1}}{\longrightarrow}\left\{\tilde{A}^{ \pm}, B^{ \pm}, \tilde{C}^{ \pm}\right\}, \\
& \left\{A^{ \pm}, B^{ \pm}, C^{ \pm}\right\} \stackrel{I_{2}}{\longrightarrow}\left\{A^{ \pm}, \tilde{B}^{ \pm}, \tilde{C}^{\mp}\right\} .
\end{aligned}
$$

The set $\left\{A^{ \pm}, \tilde{A}^{ \pm}, B^{ \pm}, \tilde{B}^{ \pm}, C^{ \pm}, \tilde{C}^{ \pm}, A, \tilde{A}, B, \tilde{B}, C, \tilde{C}\right\}$ closes a Lie algebra of rank 3: so(6). However, instead of the six non-independent generators $A, \tilde{A}, B, \tilde{B}, C, \tilde{C}$ it is enough to consider three independent diagonal operators $L_{0}, L_{1}, L_{2}$ defined by $L_{i} \Psi_{\left(l_{0}, l_{1}, l_{2}\right)} \equiv l_{i} \Psi_{\left(l_{0}, l_{1}, l_{2}\right)}$. The Hamiltonian can be expressed in terms of the so(6)-Casimir operator by means of the 'symmetrization' of the $u(3)$-Hamiltonian (22)

$$
\begin{aligned}
H= & \left\{A^{+}, A^{-}\right\}+\left\{B^{+}, B^{-}\right\}+\left\{C^{+}, C^{-}\right\}+\left\{\tilde{A}^{+}, \tilde{A}^{-}\right\}+\left\{\tilde{B}^{+}, \tilde{B}^{-}\right\}+\left\{\tilde{C}^{+}, \tilde{C}^{-}\right\} \\
& +L_{0}{ }^{2}+L_{1}{ }^{2}+L_{2}{ }^{2}+\frac{41}{12} .
\end{aligned}
$$

The intertwining generators of $s o(6)$ give rise to larger 3-dimensional Hamiltonian hierarchies

$$
\left\{H_{\left(l_{0}+m+p, l_{1}+m-n-p, l_{2}+n\right)}\right\}, \quad m, n, p \in \mathbb{Z},
$$

each one including a class of the previous ones coming from $u(3)$.

The eigenstates of these $s o(6)$-hierarchies can be classified in terms of so(6) representations whose fundamental states $\Phi_{\ell}^{0}$ are determined by

$$
A^{-} \Phi_{\ell}^{0}=\tilde{A}^{-} \Phi_{\ell}^{0}=C^{-} \Phi_{\ell}^{0},
$$

and whose explicit expressions are

$$
\Phi_{\ell}^{0}(\theta, \phi)=N \cos ^{1 / 2} \theta \sin ^{1 / 2} \theta \cos \phi \sin ^{l_{2}+1 / 2} \phi .
$$

They are characterized by the eigenvalues of the diagonal operators $L_{i}$

$$
L_{0} \Phi_{\ell}^{0}=L_{1} \Phi_{\ell}^{0}=0, \quad L_{2} \Phi_{\ell}^{0}=n \Phi_{\ell}^{0}, \quad n \in \mathbb{Z}^{+} .
$$

We obtain two classes of symmetric IUR,s of so(6) that according to (24) we summarize as

$$
\begin{array}{llll}
\text { even IURs : } l_{0}=0, l_{1}=0, l_{2}=0, & \left\{H_{(m+p, m-n-p, n)}\right\}, & q m, n, p \in \mathbb{Z}, \\
\text { odd IURs : } l_{0}=0, l_{1}=0, l_{2}=1, & \left\{H_{(m+p, m-n-p, 1+n)}\right\}, & m, n, p \in \mathbb{Z} .
\end{array}
$$



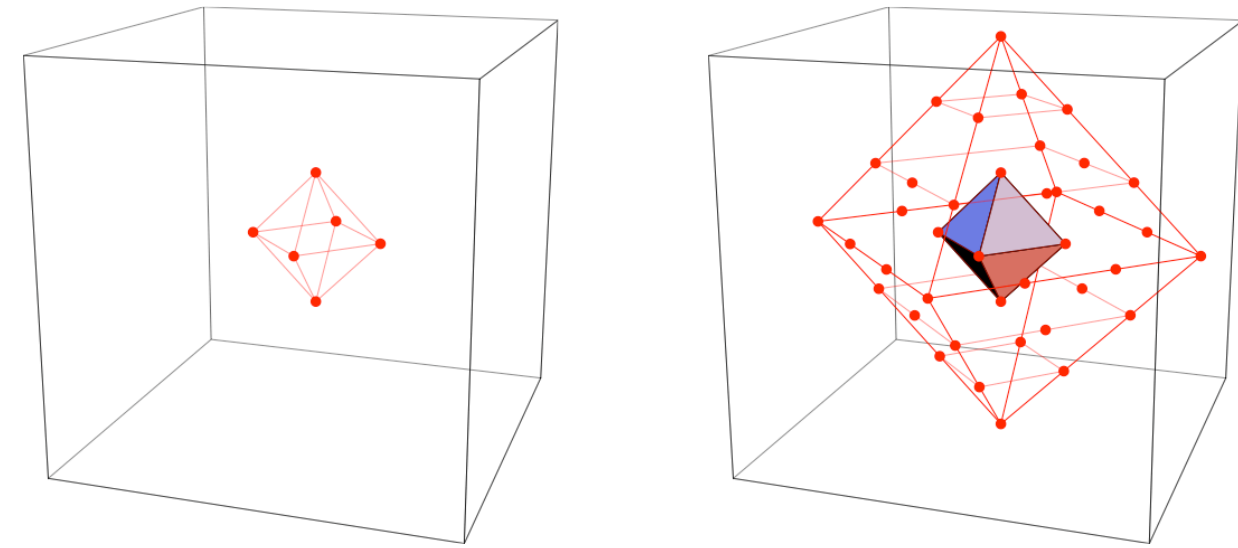

Figure 3. The points represent the states of two IUR's of $s o(6)$ with $q=1$ (left) and $q=3$ (right). The points corresponding to $q=3$ include those of $q=1$ (the inner octahedron) which are double degenerated.
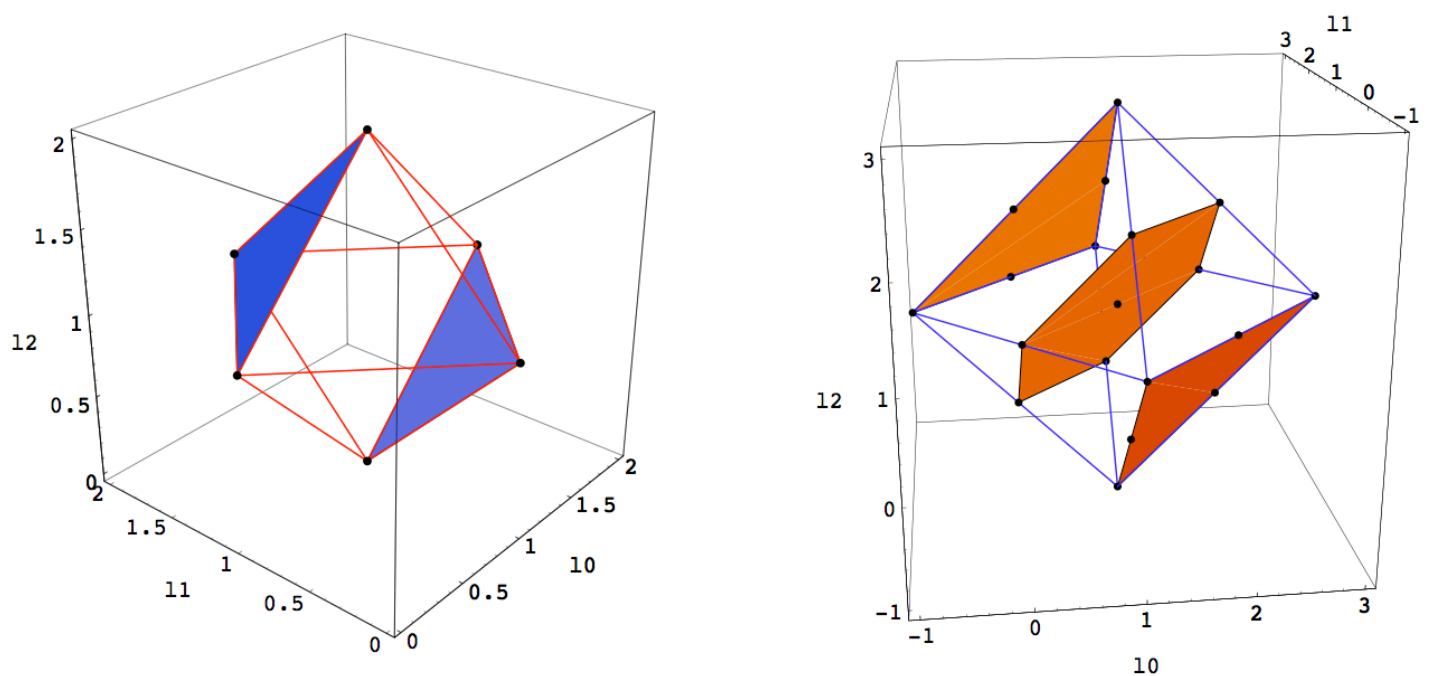

Figure 4. (Left) $q=1$ IUR of $s o(6)$ where the triangular opposite faces correspond to two IUR's of $s u(3)$. (Right) Points of a $q=2$ IUR of $s o(6)$ that are associated to three IUR's of $s u(3)$.

These representations depicted in the parameter space correspond to octahedrons, that contain iso-energy representations of $s u(3)$ labeled by $(m, n)$ such that $m+n=q$ is fixed. In Fig. 3 we represent two IUR's of so(6) characterized by $q=1$ and $q=3$, respectively. The 6 $(q=1)$-eigenstates have energy $E=\frac{5}{2} \cdot \frac{7}{2}$ and the $50(q=3)$-eigenstates share energy $E=\frac{7}{2} \cdot \frac{9}{2}$.

In Fig. 4 we show how the IUR's of $s o(6)$ corresponding to $q=1$ and $q=2$ include IUR's of $s u(3)$. Thus, in the case $q=1$ the $(m, n)$-IUR's involved are $(1,0)$ and $(0,1)$ : the two parallel exterior faces of the octahedron faces that lie on the plane characterized by $m-n$. In the other case $q=2$, the $s u(3)$-IUR's are $(2,0),(1,1)$ and $(0,2)$. The first and the last ones correspond to the opposite parallel faces of the octahedron, the $(1,1)$-IUR is represented in the parallel hexagonal section containing the origin.

\section{Conclusions}

In this section we will enumerate a list of interesting features coming from the analysis of the IO's associated to a SHS, for instance, by means of the example defined on a two-sheet hyperboloid. Obviously, from the Hamiltonian living in the sphere we can arrive to the same conclusions. 
The IO's of a SHS close an algebraic structure, in this case a non-compact $s u(2,1)$ Lie algebra. By using the reflections operators of the system we can implement these IO's obtaining a broader algebra: so $(4,2)$. These IO's lead to hierarchies of Hamiltonians described by points on planes $(s u(2,1))$ or in the 3 -dimensional space $(s o(4,2))$, corresponding to the rank of the respective algebra. This framework of IO's can be very helpful in the characterization of a physical system by selecting separable coordinate systems and determining the eigenvalues and building eigenfunctions.

We have shown the relation of eigenstates and eigenvalues with unitary representations of the $s u(2,1)$ and $s o(4,2)$ Lie algebras. In particular, we have studied the degeneration problem as well as the number of bound states. Here, we remark that such a detailed study for a 'noncompact' superintegrable system had not been realized till now, up to our knowledge. We have restricted to IUR's, but a wider analysis can be done for hierarchies associated to representations with a not well defined unitary character.

The IO's can also be used to find the second order integrals of motion for a Hamiltonian $H_{\ell}$ and their algebraic relations, which is the usual approach to (super)-integrable systems. However, we see that it is much easier to deal directly with the IO's, which are more elementary and simpler, than with constants of motion. The second and third order constants of motion close a quadratic algebra.

By means of the IO technique we have recovered the algebraic structure of the system that was used in the Marsden-Weinstein reducing procedure

$$
s u(p, q) \stackrel{\mathrm{M}-\mathrm{W}}{\longrightarrow} s o(p, q) \stackrel{\text { factoriz. }}{\longrightarrow} u(p, q) \stackrel{\text { discrete }}{\longrightarrow} s o(2 p, 2 q) .
$$

This is a step to confirm the conjecture by Grabowski-Landi-Marmo-Vilasi [29] that "any completely integrable system should arise as reduction of a simpler one (associated for instance to a simple Lie algebra)".

Our program in the near future is the application of this method to wider situations. For example, to be useful when dealing with other SHS, but not necessarily maximally integrable, or not having a system of separable variable but still allowing algebraic methods.

\section{Acknowledgments}

This work has been partially supported by DGES of the Ministerio de Educación y Ciencia of Spain under Project FIS2005-03989 and Junta de Castilla y León (Spain) (Project GR224).

\section{References}

[1] Evans N.W., Superintegrability in classical mechanics, Phys. Rev. A 41 (1990), 5666-5676.

Evans N.W., Super-integrability of the Winternitz system, Phys. Lett. A 147 (1990), 483-486.

Evans N.W., Group theory of the Smorodinsky-Winternitz system, J. Math. Phys. 32 (1991), 3369-3375.

[2] Bonatsos D., Daskaloyannis C., Kokkotas K., Deformed oscillator algebras for two-dimensional quantum superintegrable systems, Phys. Rev. A 50 (1994), 3700-3709, hep-th/9309088.

[3] Daskaloyannis C., Quadratic Poisson algebras of two-dimensional classical superintegrable systems and quadratic associative algebras of quantum superintegrable systems, J. Math. Phys. 42 (2001), 1100-1119, math-ph/0003017.

[4] Grosche C., Pogosyan G.S., Sisakian A.N., Path integral discussion for Smorodinsky-Winternitz potentials. II. The two- and three-dimensional sphere, Fortschr. Phys. 43 (1995), 523-563, hep-th/9402121.

Grosche C., Pogosyan G.S., Sisakian A.N., Path integral approach to superintegrable potentials. III. Twodimensional hyperboloid, Phys. Particles Nuclei 27 (1996), 244-272.

Grosche C., Pogosyan G.S., Sisakian A.N., Path integral discussion for superintegrable potentials. IV. Three dimensional pseudosphere, Phys. Particles Nuclei 28 (1997), 486-519.

[5] Ballesteros A., Herranz F.J., Santander M., Sanz-Gil T., Maximal superintegrability on $N$-dimensional curved spaces, J. Phys. A: Math. Gen. 36 (2003), L93-L99, math-ph/0211012. 
[6] Cariñena J.F., Rañada M.F., Santander M., Central potentials on spaces of constant curvature: the Kepler problem on the two-dimensional sphere $\mathcal{S}^{2}$ and the hyperbolic plane $\mathcal{H}^{2}$, J. Math. Phys. 46 (2005), 052702, 25 pages, math-ph/0504016.

Cariñena J.F., Rañada M.F., Santander M., Superintegrability on curved spaces, orbits and momentum hodographs: revisiting a classical result by Hamilton, J. Phys. A: Math. Theor. 40 (2007), 13645-13666.

[7] Lakshmanan M., Eswaran K., Quantum dynamics of a solvable nonlinear chiral model, J. Phys. A: Math. Gen. 8 (1975), 1658-1669.

[8] Higgs P.W., Dynamical symmetries in a spherical geometry. I, J. Phys. A: Math. Gen. 12 (1979), 309-323.

[9] Marsden J.E., Weinstein A., Reduction of symplectic manifolds with symmetry, Rep. Math. Phys. 5 (1974), $121-130$.

[10] del Olmo M.A., Rodríguez M.A., Winternitz P., Integrable systems based on $\mathrm{SU}(p, q)$ homogeneous manifolds, J. Math. Phys. 34 (1993), 5118-5139.

[11] del Olmo M.A., Rodríguez M. A., Winternitz P., The conformal group $\mathrm{SU}(2,2)$ and integrable systems on a Lorentzian hyperboloid, Fortschr. Phys. 44 (1996), 199-233, hep-th/9407080.

[12] Calzada J.A., del Olmo M.A., Rodríguez M.A., Classical superintegrable $\mathrm{SO}(p, q)$ Hamiltonian systems, J. Geom. Phys. 23 (1997), 14-30.

[13] Calzada J.A., del Olmo M.A., Rodríguez M.A., Pseudo-orthogonal groups and integrable dynamical systems in two dimensions, J. Math. Phys. 40 (1999), 188-209, solv-int/9810010.

[14] Calzada J.A., Negro J., del Olmo M.A., Rodríguez M.A., Contraction of superintegrable Hamiltonian systems, J. Math. Phys. 41 (1999), 317-336.

[15] Matveev V.B., Salle M.A., Darboux transformations and solitons, Springer Series in Nonlinear Dynamics, Springer-Verlag, Berlin, 1991.

[16] Alhassid Y., Gürsey F., Iachello F., Group theory approach to scattering, Ann. Physics 148 (1983), 346-380.

[17] Kuru Ş., Tegmen A., Vercin A., Intertwined isospectral potentials in an arbitrary dimension, J. Math. Phys. 42 (2001), 3344-3360, quant-ph/0111034.

[18] Demircioglu B., Kuru Ş., Önder M., Vercin A., Two families of superintegrable and isospectral potentials in two dimensions, J. Math. Phys. 43 (2002), 2133-2150, quant-ph/0201099.

[19] Fernández D.J., Negro J., del Olmo M.A., Group approach to the factorization of the radial oscillator equation, Ann. Physics 252 (1996), 386-412.

[20] Infeld L., Hull T.E., The factorization method, Rev. Modern Phys. 23 (1951), 21-68.

[21] Calzada J.A., Negro J., del Olmo M.A., Superintegrable quantum $u(3)$ systems and higher rank factorizations, J. Math. Phys. 47 (2006), 043511, 17 pages, math-ph/0601067.

[22] Calzada J.A., Kuru Ş., Negro J., del Olmo M.A., Intertwining symmetry algebras of quantum superintegrable systems on the hyperboloid, J. Phys. A: Math. Theor. 41 (2008), 255201, 11 pages, arXiv:0803.2117.

[23] Kobayashi S., Nomizu K., Foundations of differential geometry, Interscience Publishers, New York - London, 1963.

[24] del Olmo M.A., Rodríguez M.A., Winternitz P., Zassenhaus H., Maximal abelian subalgebras of pseudounitary Lie algebras, Linear Algebra Appl. 135 (1990), 79-151.

[25] Zhedanov A.S., The "Higgs algebra" as a "quantum" deformation of SU(2), Modern Phys. Lett. A 7 (1992), $507-512$.

[26] Bambah B.A., Sunil Kumar V., Mukku C., Polynomial algebras: their representations, coherent states and applications to quantum mechanics, J. Theor. Phys. Group Theory Nonlinear Opt. 11 (2007), 265-284.

[27] Kalnins E.G., Miller W. Jr., Pogosyan G.S., Superintegrability and associated polynomial solutions: Euclidean space and the sphere in two dimensions, J. Math. Phys. 37 (1996), 6439-6467.

[28] Kalnins E.G., Miller W. Jr., Pogosyan G.S., Superintegrability on the two-dimensional hyperboloid, J. Math. Phys. 38 (1997), 5416-5433.

[29] Grabowski J., Landi G., Marmo G., Vilasi G., Generalized reduction procedure: symplectic and Poisson formalism, Fortschr. Phys. 42 (1994), 393-427, hep-th/9307018. 